\title{
1 Discovery of bactericides as an acute mitochondrial membrane damage inducer
}

2 Running title; Chemical-biological tool of mitochondrial membrane damage

3 Ryan Houston ${ }^{1}$, Yusuke Sekine ${ }^{1,2}$, Mads B Larsen ${ }^{1}$, Kei Murakami ${ }^{3}$, Derek P Narendra ${ }^{4}$, Bill B Chen ${ }^{1,5,6}$, 4 and Shiori Sekine , $^{1 *}$

6 1. Aging Institute, Department of Medicine, School of Medicine, University of Pittsburgh, Pittsburgh, PA,

$7 \quad$ USA

8 2. Division of Endocrinology and Metabolism, Department of Medicine, School of Medicine, University

9 of Pittsburgh, Pittsburgh, PA, USA

10 3. Department of Chemistry, School of Science, Kwansei Gakuin University, Sanda, Hyogo, Japan

11 4. Inherited Movement Disorders Unit, Neurogenetics Branch, National Institute of Neurological

12 Disorders and Stroke, National Institutes of Health, Bethesda, MD, USA

13 5. Acute Lung Injury Center of Excellence, Division of Pulmonary, Allergy, and Critical Care Medicine,

14 Department of Medicine, School of Medicine, University of Pittsburgh, Pittsburgh, PA, USA

15 6. Vascular Medicine Institute, Department of Medicine, School of Medicine, University of Pittsburgh,

16 Pittsburgh, PA, USA

17 7. Division of Cardiology, Department of Medicine, School of Medicine, University of Pittsburgh,

18 Pittsburgh, PA, USA

$21 *$ Corresponding author

22 Shiori Sekine

23 Mailing address: Bridgeside Point 1, Room 568, 100 Technology Drive, Pittsburgh, PA 15219

24 Phone: 412-383-0867

25 Email: sekine@pitt.edu 


\section{Abstract}

2 Mitochondria evolved from endosymbiotic bacteria to become essential organelles of eukaryotic cells.

3 The defined lipid composition and structure of mitochondrial membranes are critical for the proper

4 functioning of mitochondria. However, mitochondrial stress responses that help maintain the integrity of

5 mitochondrial membranes against internal or external insults are not well understood. One reason for this

6 lack of insight is the absence of efficient tools to specifically damage mitochondrial membranes. Here,

7 through a compound screen originally aimed at identifying inhibitors of the inner mitochondrial

8 membrane (IMM)-resident protease OMA1, we found that two bis-biguanide compounds, Chlorhexidine

9 and Alexidine, modified OMA1 activity by altering the integrity of the IMM. Interestingly, these

10 compounds are well-known bactericides whose mechanism of action has centered on their damage-

11 inducing activity on bacterial membranes. We found Alexidine binds to the IMM likely through the

12 electrostatic interaction driven by the membrane potential as well as an affinity for anionic phospholipids.

13 Electron microscopic analysis revealed that Alexidine severely perturbated the IMM, especially the

14 cristae structure. Along with this, we observed the altered localization of IMM-resident membrane-

15 shaping proteins, including Mic60. Notably, Alexidine evoked a specific transcriptional/proteostasis

16 signature that was not induced by other typical mitochondrial stressors, highlighting the unique property

17 of Alexidine as a novel mitochondrial membrane stressor. Our findings provide a chemical-biological tool

18 that can induce acute and selective perturbation of the IMM integrity, which should enable the delineation

19 of mitochondrial stress-signaling pathways required to maintain the mitochondrial membrane homeostasis. 


\section{Introduction}

2 Mitochondria are essential and multi-functional organelles of the cell that are involved in energy

3 production, metabolic processes and cellular signaling. Evolutionally, mitochondria evolved from $\alpha$ -

4 proteobacteria that invaded into host eukaryotic cells. Mitochondria are surrounded by two membranes;

5 the outer mitochondrial membrane (OMM) and the inner mitochondrial membrane (IMM). Both

6 membranes have a characteristic phospholipid composition and structure. Reflecting the endosymbiotic

7 origin of mitochondria, the IMM shares similarity with the lipid composition of bacterial membranes

8 including high levels of cardiolipin (CL) [1-3]. CL is a non-bilayer forming phospholipid that destabilizes

9 the lipid order in bilayers and induces high membrane curvature. Together with

10 phosphatidylethanolamine (PE), which has similar biophysical properties as CL, these non-bilayer lipids

11 make up approximately $\sim 50 \%$ of the phospholipids in the IMM and are more abundant in this membrane

12 than in any other cellular membranes [1-3]. CL and PE contribute to the characteristic highly-folded

13 structure of the IMM as represented by cristae, the concave membrane structure of the IMM [4]. Among

14 the biological membranes in the cells, the IMM is known to possess the highest protein density, allowing

15 various essential bioenergetic reactions to occur. The activities of the IMM-embedded enzymes, including

16 the OXPHOS proteins, rely on the defined lipid composition and structures of the IMM [4]. Therefore,

17 the integrity of the IMM must be carefully monitored and maintained in the face of internal or external

18 insults.

19 Various mitochondrial stress responses that maintain healthy mitochondrial network have been

20 discovered [5]. These include mitophagy, an autophagic degradation of damaged mitochondria [6], as

21 well as the mitochondrial unfolded protein response (mtUPR) [7], that up-regulates a specific

22 transcriptional program to relieve mitochondrial proteotoxic stress. Importantly, the existence of small

23 molecules that can mimic a distinct type of mitochondrial damage has significantly contributed to the

24 discovery and understanding of these crucial mitochondrial stress-signaling pathways. For example, the

25 mitochondrial protonophore carbonyl cyanide m-chlorophenyl hydrazone (CCCP) and the combination of

26 OXPHOS inhibitors, Antimycin and Oligomycin, have been widely used for the mechanistic analysis of 
1 PINK1/Parkin-mediated mitophagy [8-10]. The treatment of the cells with these compounds results in

2 mitochondrial membrane potential loss, one of the hallmarks of OXPHOS dysfunction, triggering

3 mitophagy. In mammalian cells, the mtUPR can be induced by CDDO, an inhibitor of the matrix-resident

4 protease LONP, or GTPP1, a mitochondrial HSP90 inhibitor [11]. Moreover, mitochondrial proteotoxic

5 stress can be induced by Actinonin [12, 13], an inducer of mito-ribosome stalling that results in the

6 blockade of mitochondrial protein translation. A series of mitochondrial import blockers, so-called

7 MitoBloCKs, can impair mitochondrial protein import pathways [14, 15]. Recent omics analyses of the

8 mammalian cells that were treated with these mitochondrial stressors revealed that many of these stressors

9 commonly activate the integrated stress response (ISR) [16]. The ISR induces the expression of particular

10 cytoprotective genes through the activation of the transcription factor (ATF4), suggesting the existence of

11 an intimate mitochondria-nuclear communication to activate the proper stress response following specific

12 mitochondrial stressors. Recent studies using cell genetic screens for genes involved in the mitochondrial

13 stressor-induced ISR revealed that mitochondrial proteolysis plays a critical role in activating the ISR [17,

14 18]. These examples clearly highlight the importance of chemical compounds that can induce a specific

15 mitochondrial stress in identifying and analyzing mitochondrial stress-signaling pathways.

16 Recent identification of sets of lipid synthesis enzymes and lipid transfer proteins significantly

17 advanced our understanding of lipid metabolism within mitochondria [2, 3]. However, it is still not

18 known how mitochondrial membrane homeostasis is preserved under conditions that disturb

19 mitochondrial membrane integrity. This is partly due to a lack of established compounds that can

20 specifically perturb the phospholipid environment of mitochondrial membranes.

21 Here, through our unbiased small-compound screen that targeted the IMM-integral protease

22 OMA1, we found that two small-compounds, Chlorhexidine and Alexidine, that acutely disrupted the

23 integrity of mitochondrial membranes and thereby secondarily alter OMA1 activity. Interestingly, these

24 compounds are known as bactericides that have damage-inducing activities on bacterial membranes. Our

25 biochemical analyses revealed that Alexidine had an affinity to mitochondrial membranes and particularly

26 damage the cristae membranes in the IMM. Moreover, we found that the Alexidine-treatment induced 
1 characteristic transcriptional and proteostatic signatures that were not observed with other typical

2 mitochondrial stressors. Our discovery therefore provides a unique chemical-biological tool that can

3 acutely and selectively perturb membrane homeostasis in the IMM.

4

5

6

7

8

9

10

11

12

13

14

15

16

17

18

19

20

21

22

23

24

25

26 


\section{Results}

\section{Compound screen of OMA1 inhibitors identifies bactericides.}

In heathy mitochondria which maintain mitochondrial membrane potential, PTEN-induced kinase

51 (PINK1) is cleaved by the IMM-resident protease PARL just after the mitochondrial import of PINK1

6 [19] (Supplementary Fig. 1A, left panel). The cleaved product of PINK1 is retro-translocated into the

7 cytosol for proteasomal degradation [20]. In contrast, mitochondrial depolarization induces the

8 mitochondrial import arrest of PINK1, which results in the accumulation of the full-length form of PINK1

9 and its kinase activation on the OMM of damaged mitochondria [9, 21, 22] (Supplementary Fig. 1A,

10 middle panel). Activated PINK1 promotes the autophagic elimination of damaged mitochondria, so-called

11 mitophagy, cooperatively working with the cytosolic E3 ligase Parkin [6]. Mutations in PINK1 or Parkin

12 cause recessive early-onset Parkinson's disease (PD), suggesting a protective role of mitophagy in PD

13 pathogenesis [6]. We have previously reported that several PD-related PINK1 mutants are insensitive to

14 the mitochondrial stress-dependent import arrest and fail to accumulate in the OMM [23, 24]. While these

15 PINK1 mutants are cleaved by PARL in a similar way to PINK1 wild-type (WT) in healthy mitochondria,

16 the mis-sorted PINK1 mutants in depolarized mitochondria are instead cleaved by another IMM protease

17 OMA1 and are subsequently subjected to proteasomal degradation [23] (Supplementary Fig. 1A, right

18 panel). PINK1 (C125G) is one of these import arrest-defective PD-related PINK1 mutants. We stably

19 expressed PINK1 (C125G)-EYFP in PINK1 knockout (KO) cells and confirmed our previous findings by

20 immunoblotting. PINK1 protein was not observed under steady state conditions (Fig. 1A, lane 1 and 4).

21 Treatment with the proteasome inhibitor MG132, however, resulted in the accumulation of the cleaved

22 form of PINK1 (C125G)-EYFP (Fig. 1A, lane 3 and 6), indicating constitutive proteasomal degradation

23 of PINK1 (C125G)-EYFP after PARL-mediated cleavage in healthy mitochondria. Importantly, PINK1

24 (C125G)-EYFP could not accumulate in response to CCCP in control siRNA-treated cells (Fig. 1A, lane

25 2), while the full-length form of PINK1 (C125G)-EYFP did accumulate when OMA1 expression was 
1 suppressed by an OMA1-specific siRNA (Fig. 1A, lane 5). Thus, in depolarized mitochondria, PINK1

2 (C125G)-EYFP is degraded through OMA1.

3 We realized this assay system might allow for the discovery of OMA1 inhibitors since the level

4 of PINK1 (C125G)-EYFP under depolarized conditions was dependent on OMA1 activity. We therefore

5 next measured the intensity of the YFP signal of PINK1 (C125G)-EYFP after CCCP-treatment using a

6 high-content image analyzer. The Z'-factor (a factor that can evaluate the effectiveness of a high-

7 throughput screening) calculated by the YFP signal intensity derived from negative control siRNA vs.

8 OMA1 siRNA yielded a significantly high value (Z'-factor=0.74) (Supplementary Fig. 1B), indicating the

9 potential robustness of our screening system ( $Z^{\prime}<0.0$, not a suitable assay; $0.0<Z^{\prime}<0.5$, a marginal assay;

$100.5<Z^{\prime}<1.0$, an excellent assay; $Z^{\prime}=1.0$, ideal assay) [25]. The initial screen was performed with an FDA-

11 approved library consisting of approximately 1,100 compounds (Supplementary table 1). The top 3 hit

12 compounds that induced the accumulation of PINK1 (C125G)-EYFP were proteasome inhibitors (Fig. 1B

13 and Supplementary table 1), confirming the degradation of PINK1 (C125G)-EYFP occurs through

14 proteasome. Among several other hit compounds, we focused on Chlorhexidine and Chlorhexidine

15 hydrochloride, which were independently identified within top 10 hits (Fig. 1B and Supplementary table

16 1). Chlorhexidine is a bis-biguanide compound (Fig. 1C) that clinically is used as a bactericide,

17 particularly in handwashing and oral care products [26, 27]. There is a similar bis-biguanide compound

18 called Alexidine (Fig. 1D) [26]. These compounds share structural similarities in that they contain

19 symmetrical biguanide units tethered by a long alkyl chain. Strikingly, the treatment with Chlorhexidine

20 or Alexidine, but not CCCP, significantly promoted PINK1 (C125G) accumulation in a dose- and time-

21 dependent manner (Fig. 1E and F). While both Chlorhexidine and Alexidine lowered the mitochondrial

22 membrane potential similar to CCCP (Supplementary Fig. 1C), these observations suggest a membrane

23 depolarization-independent mechanism for the PINK1 (C125G) accumulation by these bactericides.

24 Alexidine was chosen for further study, as it showed a stronger stabilization activity for PINK1 (C125G)

25 than did Chlorhexidine. Alexidine, but not CCCP, induced the accumulation of PINK1 (C125G) on 
1 mitochondria (Fig. 1G). These results suggest that the identified bactericides somehow prevented OMA1-

2 mediated PINK1 (C125G) degradation.

\section{Alexidine demonstrates a substrate-dependent inhibition of OMA1-mediated proteolysis}

OMA1 is a stress-responsive protease whose proteolytic activity is enhanced in response to mitochondrial damage including CCCP-induced mitochondrial depolarization [28, 29]. OMA1 activation is achieved by its self-cleavage that eventually leads to the complete degradation of OMA1 [30, 31]. Thus,

9 inhibit the OMA1-mediated degradation of PINK1 (C125G) (Fig. 1E and F, PINK1 panels), we noticed

10 that the autocatalytic degradation of OMA1 was enhanced in response to these bactericides as observed

11 under CCCP-treated conditions (Fig. 1E and F, OMA1 panels). Our previous report suggested that the

12 OMA1 inhibition not only prevented the degradation of PINK1 (C125G) but also restored its kinase 13 activity and thus the subsequent mitochondrial recruitment of Parkin [23]. However, treatment with

14 Alexidine did not restore the kinase activity of PINK1 (C125G) (Supplementary Fig. 1D, lane 7 in a 15 Phos-tag gel).

These results raised the possibility that the bactericides-mediated stabilization of PINK1 (C125G)

17 cannot simply be attributed to the inhibition of OMA1 proteolytic activity. Therefore, we examined the

18 cleavage of other known OMA1 substrates following Alexidine treatment. We tested the dynamin-like

19 GTPase OPA1 [28, 29], and the mitochondrial protein phosphatase PGAM5 [32, 33]. It is known that

20 both of these mitochondrial proteins are cleaved in response to CCCP. While the CCCP-induced OPA1

21 cleavage mostly depends on OMA1 [28, 29], the CCCP-induced PGAM5 cleavage redundantly depends

22 on both OMA1 and PARL $[32,33]$. To examine the effect of Alexidine on the OMA1-mediated PGAM5

23 cleavage, we tested whether Alexidine pre-treatment could inhibit CCCP-dependent PGAM5 cleavage in

24 PARL KO cells. We observed that in PARL KO cells, Alexidine pre-treatment inhibited CCCP-induced 25 PGAM5 cleavage in a time- and dose-dependent manner (Fig. 1H and Supplementary Fig. 1E, PGAM5 26 panels). In contrast, CCCP-dependent OPA1 cleavage was still observed even with Alexidine pre- 
1 treatment (Fig. 1H and Supplementary Fig. 1E, OPA1 panels). These results suggest that Alexidine

2 prevented OMA1-mediated PGAM5 cleavage, but not OMA1-mediated OPA1 cleavage. Recently, three 3 other OMA1 substrates were reported; CHCHD2, CHCHD10 and DELE1. CHCHD2 and CHCHD10 are

4 degraded by OMA1 after treatment with Actinonin, a mitochondrial translation inhibitor [34]. DELE1 is

5 cleaved by OMA1 in response to mitochondrial damage including CCCP, which induces the ISR through

6 activation of HRI, one of the eIF2 $\alpha$ kinases [17, 18]. Again, Alexidine showed a distinct effect on

7 OMA1-mediated proteolysis depending on the particular substrate. Alexidine pre-treatment inhibited

8 OMA1-mediated degradation of CHCHD2 and CHCHD10 (Fig. 1I), while it failed to inhibit OMA1-

9 mediated cleavage of DELE1 (Fig. 1J). Collectively, these results suggest that Alexidine is not a simple

10 OMA1 inhibitor (rather, it activates OMA1 itself), but it inhibits the OMA1-mediated proteolysis in a

11 substrate-dependent manner (Fig. 1K).

\section{Alexidine has an affinity for the IMM.}

We next tried to identify a target of Alexidine to address the underlying molecular mechanism of

15 the observed substrate-specific action of Alexidine on OMA1-mediated proteolysis. In addition to 16 antimicrobial properties, Chlorhexidine and Alexidine are also reported as inhibitors of PTPMT1 [35], a 17 mitochondrial matrix-localized phosphatase that dephosphorylates phosphatidylglycerol-phosphate (PGP), 18 an essential intermediate in cardiolipin (CL) biosynthesis [36, 37]. However, knockdown (KD) of 19 PTPMT1 in our PINK1 (C125G)-EYFP stable HeLa cells did not promote PINK1 (C125G) stabilization

20 (Supplementary Fig. 2, lane 5), suggesting that Alexidine appears to have a different target in this context.

The proposed mechanism of action of Chlorhexidine as a bactericide centers on its bacterial

22 membrane damage-inducing ability through its interaction with phospholipids [26, 27]. Alexidine also has

23 a similar activity on the bacterial membranes [26]. Guanidinium groups of these compounds possess

24 delocalized positive charges at physiological pH [38] (Fig. 1C and D). The delocalized positive charges

25 have higher lipophilicity compared with groups that have localized charges, which is considered to confer

26 the efficient binding ability of Chlorhexidine and Alexidine to phospholipids, together with their long 
1 alkyl chain between two symmetric guanidinium groups [26, 27]. These observations led us to examine

2 the effect of Alexidine on the phospholipids in the IMM. We first tested a phospholipid dye 10-N-Nonyl

3 acridine orange (NAO) staining with or without Alexidine-treatment. NAO is a lipophilic and positively

4 charged molecule that is often utilized to stain CL in mitochondria $[39,40]$, and is also to monitor anionic

5 phospholipids in bacterial membranes [41]. In eukaryotic cells, it is widely known that NAO selectively

6 accumulates in the IMM $[39,40]$. We found that treatment with Alexidine but not CCCP dramatically

7 reduced mitochondrial NAO staining (Fig. 2A). NAO staining was rapidly lost after drug-treatment (Fig.

8 2B). In contrast, the fluorescent intensity of Su9-mCherry (matrix marker) was not affected, indicating

9 that mitochondria themselves were still present (Fig. 2B). These results indicate that Alexidine has effects

10 on the IMM phospholipids. To directly evaluate the binding affinity of Alexidine to phospholipids, we

11 examined the effect of Alexidine on in vitro binding between NAO and anionic phospholipid species

12 coated on microplate wells [42, 43]. Pre-incubation with Alexidine was found to reduce the fluorescent

13 intensity derived from NAO bound to anionic phospholipids in a dose-dependent manner (Fig. 2C).

14 Consistent with the NAO staining in cells (Fig. 2A), CCCP did not reduce NAO intensity in this assay

15 (Fig. 2C). These results suggest that Alexidine has an affinity to anionic phospholipids and competes with

16 NAO to bind to these IMM phospholipids.

17 Intriguingly, the aforementioned chemical property of guanidium groups is also often utilized to

18 target drugs to mitochondria, because it is known to preferentially accumulate in the IMM which has an

19 electrochemical gradient $[44,45]$. Therefore, we tested whether mitochondrial depolarization prevents the

20 action of Alexidine on the IMM. CCCP-pretreatment partially attenuated the Alexidine-induced reduction

21 of NAO staining (Fig. 2D and E). These results suggest that the mitochondrial membrane potential can be

22 a primary driving force for the mitochondrial targeting of Alexidine, and the high lipophilicity of

23 Alexidine promotes the accumulation of this compound in the hydrophobic lipid environment of the IMM.

\section{Alexidine induces an acute perturbation of IMM integrity.}


Since Alexidine appeared to interact with the IMM phospholipids, we investigated the effects of Alexidine on IMM structure and on IMM-shaping proteins. The IMM is structurally subdivided into two domains; the inner boundary membrane (IBM) where the IMM is in close proximity with the OMM, and

4 the cristae, bag-like structures where the IMM invaginates into the matrix $[4,46]$. These two IMM 5 domains are connected by narrow, neck-like structures called cristae junctions (CJs) [4, 46] (Fig. 3A). To 6 examine IMM structure, we performed an electron microscopic (EM) analysis of mitochondria with or 7 without Alexidine-treatment. The EM images clearly revealed that the IMM structure was severely 8 disrupted after Alexidine-treatment (Fig. 3A, right panels). The alteration of the cristae membrane was the 9 most striking feature. The cristae membrane was pinched-off from the IBM and often appeared bunched 10 up in an onion-like ball in the matrix. Mitochondria were swollen and the matrix content seemed to be 11 diluted. However, the OMM and the IBM did not exhibit apparent morphological changes and remained 12 in place, suggesting that Alexidine may particularly influence the cristae membranes and CJs. In contrast,

13 CCCP-treatment only displayed a mild disturbance in the IMM structure (Fig. 3A, middle panels). We next examined the effects of Alexidine on proteins that help shape the IMM. The PHB 15 complex is a heteromeric complex which consists of the closely related IMM-localized proteins PHB1 and PHB2, and is predicted to form a ring-like structure in the IMM [47]. It has been shown that the PHB

17 complex may regulate the CL/PE-enriched microdomains in the IMM [48-50]. At the same time, the PHB 18 complex is also known to regulate several IMM-resident proteases including m-AAA [51], OMA1 [52, 19 53], and PARL [54]. All of these proteases are single- or multi-spanning membrane proteins, suggesting 20 the importance of the PHB complex-organized lipid micro-environments in the regulation of their 21 proteolytic activities. Because we observed substrate-specific effects of Alexidine on OMA1-mediated 22 proteolysis (Fig. 1K), we next examined the localization of OMA1 and PHB2. To prevent the stress23 dependent auto-catalytic degradation of OMA1, we stably expressed an OMA1 protease activity-dead 24 mutant, OMA1 (E328Q) [23], in OMA1 KO cells. The localization of OMA1 and PHB2 mostly 25 overlapped under steady state and CCCP-treated conditions (Fig. 3B, upper and lower panels). However, 26 under Alexidine-treated conditions, the localization of these proteins diverged, with part of the OMA1 
1 pool now segregated from the PHB2-positive IMM (Fig. 3B, middle panels). When the IMM was stained

2 with Cox IV, a subunit of the cytochrome c oxidase complex, the localization of OMA1 overlapped with

3 the Cox IV-positive IMM even under Alexidine-treated conditions (Fig. 3C, middle panels), indicating

4 that OMA1 still exists in the IMM.

The MICOS complex is located at CJs where it stabilizes membrane curvature and forms contact sites (CSs) between the OMM and the IMM [46] (Fig. 3A). It is reported that the MICOS complex genetically interact with the CL synthesis pathway $[55,56]$, and that some components (Mic60 and

8 Mic27) of the MICOS complex directly bind to CL in vitro [57, 58]. Among the 7 components of the

9 metazoan MICOS complex [46], we examined Mic60 localization before and after the Alexidine-

10 treatment. Under the resolution of conventional confocal microscopy, Mic60 shows a uniform distribution

11 along the mitochondrial string at steady state conditions (Fig. 3D, upper panels). However, after

12 Alexidine-treatment, Mic60 was localized in a restricted region of each fragmented mitochondrion and

13 showed an intense, bright, puncta-like localization within the IMM (Fig. 3D, middle panels). In contrast

14 to the Alexidine-treated cells, Mic60 was uniformly distributed in each fragmented mitochondrion after

15 CCCP-treatment (Fig. 3D, lower panels), indicating that Mic60 puncta formation is specifically induced

16 by Alexidine. Taken together, these observations suggest that Alexidine induced acute redistributions of

17 IMM-resident proteins along with the perturbation of IMM subdomain integrity.

Alexidine evokes a unique transcriptional/proteostasis signature.

From the observations above, we hypothesized that Alexidine could be used as an acute

21 mitochondrial membrane damage inducer. Therefore, we decided to characterize the cellular response

22 elicited by the Alexidine-induced mitochondrial membrane perturbation. For this purpose, we performed

23 TMT-based quantitative proteomics (Supplementary table 2). As described so far, Alexidine induced

24 mitochondrial alterations that were distinct from CCCP-treatment. In order to identify the proteins whose

25 expression was specifically changed in response to the Alexidine-induced mitochondrial membrane stress,

26 we compared three different conditions: mock, Alexidine and CCCP-treatment. Gene Ontology analysis 
1 for proteins which were significantly changed in the Alexidine-treated cells showed a significant 2 enrichment of mitochondria-related proteins (Fig. 4A), suggesting that Alexidine preferentially affects 3 mitochondria among several other organelles. Many proteins were commonly upregulated or 4 downregulated in both the Alexidine and CCCP-treated cells (Fig. 4B), which may be attributed to the 5 observation that Alexidine also induces mitochondrial depolarization at almost the same level as CCCP 6 (Supplementary Fig. 1C). Notably, the expression of some proteins was specifically altered in the 7 Alexidine-treated cells. Twenty-seven proteins were specifically identified as down-regulated proteins in 8 Alexidine-treated cells (fold change < 0.8, T-TEST q-value < 0.05), (Fig. 4C and Supplementary table 2).

9 Among these, 13 proteins were mitochondrial proteins (Fig. 4D). These include OXPHOS proteins, 10 proteins which are involved in Coenzyme Q biosynthesis, and PTPMT1. As up-regulated proteins (fold 11 change $>1.5$, T-TEST q-value $<0.05$ ), only 4 non-mitochondrial proteins were specifically up-regulated 12 in response to Alexidine (Fig. 4E and Supplementary table 2). These include metallothioneins (MTs) and 13 heme oxygenase 1 (HMOX1), an essential enzyme in heme catabolism [59] (Fig. 4F). We next sought to 14 confirm the Alexidine-specific expression changes obtained from the proteomics analysis. 15 Immunoblotting analysis confirmed that HMOX1 was up-regulated while COA7, COX17 and PTPMT1 16 were down-regulated upon Alexidine-treatment (Fig. 4G). Strikingly, these changes were only observed 17 by Alexidine-treatment, and not by other well-known mitochondrial stressors such as CCCP, Rotenone (a 18 Complex I inhibitor), Actinonin or CDDO (Fig. 4G). We confirmed that each mitochondrial stressor was 19 working in our system by monitoring the OPA1 cleavage and the OMA1 activation on immunoblotting 20 (Fig. 4G), and mitochondrial ROS production by FACS (Supplementary Fig. 3A). CDDO induced the up21 regulation of mtHSP60 transcription, one of the targets of mtUPR [11], but Alexidine did not show any 22 enhancement of mtHSP60 transcription (Supplementary Fig. 3B). RT-PCR analysis revealed that the 23 upregulation of HMOX1 and MT2A was induced at the transcriptional level (Fig. 4H), indicating the 24 existence of a mitochondrial-nuclear communication in response to Alexidine-treatment. In contrast, the 25 mRNA level of COA7 or COX17 did not change after Alexidine-treatment, indicating that Alexidine 26 likely induces the post-transcriptional degradation of these proteins (Fig. 4H). LONP knockdown 
1 prevented the Alexidine-induced downregulation of PTPMT1 (Supplementary Fig. 3C), suggesting that at

2 least PTPMT1 is degraded within the mitochondria. These results suggest that Alexidine can evoke

3 unique mitochondrial responses that are not induced by other typical mitochondrial stressors, presumably

4 through the perturbation of the IMM integrity (Supplementary Fig. 3D).

\section{Discussion}

9 In this study, we found two bactericides, Chlorhexidine and Alexidine, as small molecules that can

10 induce the acute perturbation of mitochondrial membrane integrity. EM analysis of mitochondria after

11 Alexidine-treatment showed a strikingly altered cristae membrane structure, while keeping the OMM and

12 the IBM largely in place. This suggest that Alexidine can specifically damage the cristae among several

13 distinct membrane compartments within mitochondria (Fig. 3A). It has been noted that cristae membranes

14 are the membranes where OXPHOS proteins are concentrated [4]. Our TMT-based quantitative

15 proteomics analysis indicated that many OXPHOS proteins were down-regulated after Alexidine-

16 treatment (Fig. 4D), which also supports the specific action of Alexidine on the cristae membrane.

17 Interestingly, several recent studies identified Alexidine (and Chlorhexidine) as an agent that can alter

18 cellular metabolism. This metabolic shift ultimately resulted in various effects on cells; the anti-invasive

19 and metastatic activity on tumor cells [60, 61], the maintenance of the quiescent status of stem cells [62],

20 the enhanced glucose utilization in vivo [63], or the transcription factor TFEB nuclear translocation

21 through the AMPK activation [64]. Some of these reports found that the Alexidine-treatment reduced

22 oxygen consumption [62], and preferentially shifted the energy source from OXPHOS to glycolysis [61].

23 Because PTPMT1 was reported as a metazoan target of Alexidine [35], some studies indicated above

24 speculated that the observed metabolic effects may result from the PTPMT1 inactivation. However, the 25 cristae-membrane disrupting activity of Alexidine, which we identified in this study, must now be 26 considered as a basis for the acute effects of Alexidine on the cellular metabolism. 
It still remains elusive how Alexidine specifically affects the cristae membrane. As predicted from the chemical properties of guanidium groups of Alexidine [44, 45], our NAO staining assay indicated that mitochondrial membrane potential can be a driving force for the mitochondrial targeting of Alexidine (Fig. $2 \mathrm{D}$ and E). Because of the high abundance of OXPHOS proteins in the cristae [4], the cristae membranes have higher membrane potential than the IBM [39]. This unique feature of the cristae may explain the specific effect of Alexidine on this membrane compartment within the IMM. Also, it has been suggested that the high-curvature of the cristae is created by high amounts of non-bilayer lipids such as CL and PE $[1,4]$. We demonstrated that Alexidine has a reasonable affinity for CL as it is able to compete with NAO (Fig. 2C). Therefore, this property of Alexidine may also contribute to the accumulation of Alexidine in 10 the cristae membrane.

11 As a result of Alexidine-treatment, we observed a robust induction of HMOX1, a heme-degrading 12 enzyme, and several MTs, metal chelators (Fig. 4 F-H). The direct link between the Alexidine-mediated 13 mitochondrial membrane damage and the induction of HMOX1 and MTs is not known. Early studies 14 indicate that HMOX1 and MTs are simultaneously induced by heme addition to the culture media [65]. 15 Subsequent studies have demonstrated that HMOX1 induction was mediated by nuclear factor erythroid 2-related factor 2 (NRF2), a transcription factor involved in the antioxidant response [59]. Mitochondria

17 are known as a site for heme biosynthesis [66]. Also, the mitochondrial matrix has a pool of the heavy 18 metal copper [66]. Together with Fe-S clusters that are synthesized in mitochondria, heme and copper are 19 utilized as important cofactors for various enzymes including OXPHOS proteins. Due to their harmful 20 radical-formation activity, the export of newly synthesized heme across the mitochondrial membranes is 21 tightly regulated by a membrane-embedded heme exporter, while copper chaperones ensure the safe 22 delivery of copper to target proteins [66]. However, it is possible that the Alexidine-mediated IMM 23 perturbation disturbed this regulation and resulted in the heme and copper leakage from mitochondria.

24 Ultimately, it might lead to the induction of HMOX1 and MTs as a preventive strategy. Complex IV 25 utilizes a heme-copper center to reduce oxygen [66]. We observed that the Alexidine-treatment strongly 26 degraded two Complex IV assembly factors; COA7 and COX17 [66-68] (Fig. 4G). The exact role of 
1 COA7 in the assembly of Complex IV is not known. Meanwhile, COX17 is well-known as a copper 2 chaperone that delivers copper to Complex IV [66]. Therefore, in addition to the direct leakage of heme 3 and copper from the mitochondria, it is also possible that heme and copper released from degraded 4 OXPHOS proteins activate the transcription of HMOX1 and MTs. In either case, the induction of 5 HMOX1 and MTs can be used as a sensitive marker of the mitochondrial membrane damage.

6 In addition to these transcriptional/proteostasis alterations, Alexidine remodeled the IMM-resident 7 membrane proteins including PHB2, OMA1 and Mic60 (Fig. 3 B and D). The single particle electron 8 microscopic analysis of PHB complex suggested that it forms a ring-like structure in the IMM [47]. It is 9 predicted that the ring-like PHB complex can exert a partition-like function in the IMM, where it can 10 define the lateral distribution of specific lipids, including CL and PE, or proteins such as IMM-resident 11 proteases including OMA1 [48]. As Alexidine showed an affinity to CL (Fig. 2C), Alexidine might be 12 able to accumulate in the PHB complex-organized CL/PE-enriched domain of the IMM. Previous reports 13 revealed that PHB deletion can activate OMA1 proteolytic activity without any obvious mitochondrial 14 membrane depolarization [52, 53, 69], indicating that PHB complex may hold OMA1 in the inactive state 15 presumably through restricting the protease to specific IMM microdomains. We observed the segregation of OMA1 from PHB2-positive IMM after Alexidine treatment (Fig. 3B). These observations may indicate

17 that Alexidine causes OMA1 to dissociate from the PHB complex-organized microdomain and that once 18 OMA1 is released from PHB complex-mediated inhibition, it is proteolytically active. However, OMA1 19 did not cleave or degrade certain substrates such as PINK1 (C125G), PGAM5, CHCHD2 and CHCHD10 20 upon Alexidine treatment (Fig. 1K). This observation implies that the PHB complex-organized lipid 21 microdomain not only regulates the proteolytic activity of OMA1 but may also be involved in the spatial 22 regulation of OMA1 and its substrates.

23 Mic60, one of the important components of MICOS complex [46], showed a puncta-like localization 24 within the IMM after Alexidine treatment (Fig. 3D). Among several components of MICOS complex, it 25 has been suggested that Mic60 can self-assemble and forms puncta within the IMM when all other 26 MICOS components are absent in yeast [56]. Moreover, the recent analysis showed the de novo formation 
1 of CJs by drug-controlled expression of Mic60 in reconstituted Mic60 KO cells [70], establishing a 2 critical role for Mic60 in CJ formation. Upstream determinants of Mic60 localization are not fully 3 understood. However, recent studies in yeast has revealed that the aforementioned Mic60 puncta formed

4 in the absence of other components of the MICOS complex are often observed in proximity to ER5 mitochondria contact sites, where the ERMES complex exists [71]. The ERMES complex physically

6 tethers the ER and mitochondria in yeast and creates membrane contact sites to allow efficient lipid 7 transfer between the ER and mitochondria [2]. Together with the fact that MICOS and ERMES 8 genetically interact with each other [55], it has been suggested that cooperative functions of MICOS and 9 ERMES in mitochondrial membrane architecture (ERMIONE) [72]. Mitochondrial lipid homeostasis 10 depends on both inter-organelle (mainly from the ER) and intra-organelle (between the OMM and the 11 IMM) lipid trafficking [2, 3]. Therefore, the coordinated regulation of ERMES-MICOS localization may 12 be functionally linked to allow for efficient lipid trafficking across the membranes. Although it has not 13 been examined whether the MICOS complex is involved in inter-organelle lipid trafficking, it is known 14 that the MICOS complex is involved in intra-mitochondrial lipid metabolism [57, 73]. Our EM analysis 15 revealed that the cristae structure was severely damaged following Alexidine treatment (Fig. 3A). In such 16 a case, it is expected that mitochondrial lipid demand is significantly increased in order to help restore the 17 highly-folded cristae structure. It is tempting to speculate that the Alexidine-induced Mic60 puncta 18 formation plays a role in this process as a part of a mitochondrial membrane stress response. It may be 19 also of note that several reports indicate a link between the MICOS complex and COA7 or COX17 20 (robustly degraded proteins after the Alexidine-treatment). For example, in yeast, it was shown that 21 COX17 physically interacts with Mic60 and modulates the MICOS complex formation [74]. Other 22 studies suggest that the sustained KD of Mic60 (or its interactor, Sam50) resulted in the degradation of 23 COA7 in mammalian cells [68]. COA7 was also identified as the possible interactor of Mic10, another 24 important component of MICOS complex [75]. These previous observations may explain the reason why 25 we observed the relatively specific and robust degradation of COA7 and COX17 among over 100 26 components of OXPHOS system. 
In conclusion, we discovered Alexidine and Chlorhexidine as small molecules that enable us to acutely and preferentially perturb the mitochondrial membrane architecture in the IMM. Our findings therefore offer a useful chemical-biological tool for delineating mitochondrial membrane stress responses.

$9 \quad$ Figure legends

10 Figure 1 Identification of bactericides as a substrate-dependent OMA1 inhibitor.

11 (A) PINK1 knockout (KO) HeLa cells stably expressed PINK1 (C125G)-EYFP were transfected with 12 control or OMA1 siRNA. After 72 hours, cells were treated with $20 \mu \mathrm{M}$ CCCP or $10 \mu \mathrm{M}$ MG132 for 4

13 hours. The lysate was analyzed by SDS-PAGE. (B) PINK1 KO HeLa cells stably expressed PINK1

14 (C125G)-EYFP were seeded on 384 well plate, and were treated with each compound (5 $\mu$ M, FDA-

15 approved compounds). After 18 hours, cells were treated with $20 \mu \mathrm{M}$ CCCP for 4 hours. EYFP

16 fluorescence intensity of each well was measured by the high content image analyzer. (C, D) The

17 chemical structure of a hit compound, Chlorhexidine (C), and a similar compound, Alexidine (D).

18 Guanidium groups that have delocalized positive charges are highlighted in blue. (E, F) PINK1 KO HeLa

19 cells stably expressed PINK1 (C125G)-EYFP were treated with the indicated drugs for the indicate time

20 period, and the lysate was analyzed by SDS-PAGE. (G) HeLa cells stably expressed PINK1 (C125G)-

21 EYFP were treated with $20 \mu \mathrm{M}$ CCCP or $5 \mu \mathrm{M}$ Alexidine for 8 hours, and were subjected to

22 immunocytochemistry (ICC). Tom20 was utilized as a mitochondrial marker. Scale bars; $50 \mu \mathrm{m}$. $(\mathrm{H})$

23 PARL KO HeLa cells were pre-treated with Alexidine for the indicated time period, and after, treated

24 with $20 \mu \mathrm{M}$ CCCP for 2 hours. Note that in PARL KO cells, the CCCP-dependent PGAM5 cleavage is

25 mediated by OMA1. The lysate was analyzed by SDS-PAGE. (I) WT HeLa cells were pre-treated with 5 
$1 \mu \mathrm{M}$ Alexidine for 1 hour, and after, treated with $150 \mu \mathrm{M}$ Actinonin for 2 hours. The lysate was analyzed

2 by SDS-PAGE. (J) HeLa cells transiently expressed with DELE1-HA was pre-treated with $5 \mu \mathrm{M}$

3 Alexidine for 1 hour, and after, treated with $20 \mu \mathrm{M}$ CCCP for 4 hours. The lysate was analyzed by SDS-

4 PAGE. (K) Alexidine showed the inhibitory effect on the OMA1-mediated proteolysis in a substrate-

5 dependent manner.

7 Figure 2 Alexidine has an affinity for the IMM.

8 (A and B) WT HeLa cells or HeLa cells stably expressed Su9-mCherry (a matrix marker) were treated

9 with $5 \mu \mathrm{M}$ Alexidine or $20 \mu \mathrm{M}$ CCCP for the indicated time period. After the drug-treatment, cells were

10 washed with phosphate-buffered saline (PBS) for twice, and were stained with NAO. NAO staining was

11 analyzed by live-cell imaging. Scale bars; $25 \mu \mathrm{m}$ (A) and $10 \mu \mathrm{m}$ (B). (C) NAO fluorescence intensity (Ex

$12485 \mathrm{~nm} / \mathrm{Em} 535 \mathrm{~nm}$ ) in individual wells of a microtiter plate which were coated with or without the

13 indicated phospholipid species was measured by microtiter plate reader. Alexidine or CCCP was added at

14 the indicated concentration for $30 \mathrm{~min}$ before the NAO staining. Data are shown as mean \pm S.D. ( $\mathrm{n}=3$ or

$15 \mathrm{n}=6$ per condition). $* * \mathrm{P}<0.01, * * * \mathrm{P}<0.001$, and $* * * * \mathrm{P}<0.0001$ (One-way ANOVA followed by Turkey's

16 multiple comparison). (D) WT HeLa cells were treated with $5 \mu \mathrm{M}$ Alexidine, $20 \mu \mathrm{M}$ CCCP or the

17 combination of these drugs for the indicated time period. After the drug-treatment, cells were washed

18 with phosphate-buffered saline (PBS) for twice and were stained with NAO. NAO staining was analyzed

19 by live-cell imaging. Scale bars; $50 \mu \mathrm{m}$. (E) FACS analysis of NAO fluorescence intensity in (D).

21 Figure 3 Alexidine induces an acute perturbation of IMM integrity.

22 (A) The electron microscopic (EM) images of the WT HeLa cells that were treated with DMSO, $20 \mu \mathrm{M}$

$23 \mathrm{CCCP}$, or $5 \mu \mathrm{M}$ Alexidine for 4 hours. Scale bars; $800 \mathrm{~nm}$ (upper panels), $100 \mathrm{~nm}$ (lower panels). (B, C)

24 OMA1 KO cells stably expressed OMA1 (E328Q)-EYFP were treated with $5 \mu \mathrm{M}$ Alexidine or $20 \mu \mathrm{M}$

25 CCCP for 4 hours and were subjected to ICC. Cox IV was used as a typical IMM marker protein (C). *; 
1 non-specific signal. Scale bars; $5 \mu \mathrm{m}$. (D) WT HeLa cells were treated with $5 \mu \mathrm{M}$ Alexidine or $20 \mu \mathrm{M}$

2 CCCP for 2 hours and were subjected to ICC. Tim50 was used as a typical IMM marker protein. Scale

3 bars; $5 \mu \mathrm{m}$.

Figure 4 Alexidine evokes a unique transcriptional/proteostasis signature.

(A-F) WT HeLa cells were treated with $5 \mu \mathrm{M}$ Alexidine or $20 \mu \mathrm{M}$ CCCP for 8 hours and were subjected

to TMT-based quantitative proteomics. (A) The enrichment analysis of proteins whose amount was

8 significantly changed (T-TEST q-value < 0.05) in the Alexidine-treated cells compared to the mock-

9 treated cells. The enriched Gene Ontology Cellular Component (GOCC) classes and each enrichment

10 value was shown. (B) Scatter plot for $\log _{2} \mathrm{FC}$ of protein amount in CCCP-treated/Untreated (x-axis) and

$11 \log _{2}$ FC of protein amount in Alexidine-treated/Untreated (y-axis). Values are from S2 table. (C) Venn

12 diagram of down-regulated proteins (fold change < 0.8, T-TEST q-value < 0.05) after the Alexidine or

13 CCCP-treatment. (D) List of the Alexidine-specific down-regulated mitochondrial proteins. IMS; inner

14 membrane space. (E) Venn diagram of up-regulated proteins (fold change $>1.5$, T-TEST q-value $<0.05$ )

15 after the Alexidine or CCCP-treatment. (F) List of the Alexidine-specific up-regulated proteins. $(\mathrm{G}, \mathrm{H})$

16 Validation of TMT-based proteomics results by SDS-PAGE (G) and RT-PCR (H). WT HeLa cells were

17 treated with the indicated drugs for 8 hours $(5 \mu \mathrm{M}$ Alexidine, $20 \mu \mathrm{M}$ CCCP, $10 \mu \mathrm{M}$ Rotenone, $150 \mu \mathrm{M}$

18 Actinonin, and $10 \mu \mathrm{M}$ CDDO) and were subjected to further analyses. Data are shown as mean \pm S.D.

19 ( $\mathrm{n}=3$ or $\mathrm{n}=4$ per condition) $(\mathrm{H})$.

\section{Supplementary Figure 1}

\section{Compound screen of OMA1 inhibitors identifies bactericides.}

23 (A) The mitochondrial import pathway of PINK1. See text for detail. (B) PINK1 KO HeLa cells stably

24 expressed PINK1 (C125G)-EYFP were seeded on 96 well plate and were transfected with control or

25 OMA1 siRNA (48 wells for each). After 72 hours, cells were treated with $20 \mu \mathrm{M}$ CCCP for 4 hours. Z'- 
1 factor (control siRNA vs. OMA1 siRNA) was calculated by using EYFP fluorescence intensity measured

2 by a high content image analyzer. (C) WT HeLa cells were treated with the indicated drugs for the

3 indicate time period. After each drug-treatment, mitochondrial membrane potential of each cell was

4 measured by FACS using tetramethylrhodamine-methyl ester (TMRM). (D) PINK1 KO HeLa cells stably

5 expressed with PINK1 wild-type (WT)-EYFP or PINK1 (C125G)-EYFP were treated with the indicated

6 drugs for the indicated time period. The lysate was analyzed by SDS-PAGE with or without Phos-tag. (E)

7 PARL KO HeLa cells were pre-treated with Alexidine at the indicated concentration for 30 min, and after,

8 treated with $20 \mu \mathrm{M}$ CCCP for 2 hours. Note that in PARL KO cells, the CCCP-dependent PGAM5

9 cleavage is mediated by OMA1. The lysate was analyzed by SDS-PAGE.

\section{Supplementary Figure 2}

12 Transient deletion of PTPMT1 did not promote the accumulation of PINK1 (C125G).

13 PINK1 KO HeLa cells stably expressed PINK1 (C125G)-EYFP were transfected with control, PTPMT1 14 or OMA1 siRNA. After 72 hours, cells were treated with the indicated drugs for 8 hours. The lysate was 15 analyzed by SDS-PAGE.

\section{Supplementary Figure 3}

18 Alexidine is a novel mitochondrial stressor that can induce the acute mitochondrial

19 membrane damage.

20 (A) WT HeLa cells were treated with the indicated drugs for 4 hours. After each drug-treatment, 21 mitochondrial ROS production of each cell was measured by FACS using MitoSOX. (B) WT HeLa cells

22 were treated with the indicated drugs for 4 hours $(5 \mu \mathrm{M}$ Alexidine, $20 \mu \mathrm{M}$ CCCP, $10 \mu \mathrm{M}$ Rotenone, 150

$23 \mu \mathrm{M}$ Actinonin, and $10 \mu \mathrm{M}$ CDDO) and were subjected to RT-PCR analysis. (C) WT HeLa cells were

24 transfected with the indicated control or LONP siRNAs. After 72 hours, cells were treated with $5 \mu \mathrm{M}$ 
1 Alexidine for 8 hours and were subjected to SDS-PAGE. (D) Graphical summary of this study. See text

2 for detail.

4 Supplementary Table 1

5 The results of the FDA-approved compound screen.

7 Supplementary Table 2

8 The results of the TMT-based quantitative proteomic analysis.

12 Materials and methods

\section{Cell culture, transfection, and treatments}

14 HEK293T and HeLa cells were cultured in DMEM (Gibco) supplemented with 10\% FBS (VWR Life

15 Science), $10 \mathrm{mM}$ HEPES (Gibco), $1 \mathrm{mM}$ Sodium pyruvate (Gibco), non-essential amino acids (Gibco)

16 and GlutaMAX (Gibco). Following HeLa KO cell lines were kindly provided by Dr. Richard J Youle

17 (NIH, NINDS); PINK1 KO [76], OMA1 KO [23], and PARL KO [23]. For RNA interference, 20 nM

18 Stealth siRNAs (Steath RNAi Negative Control Med GC Duplex \#2, 12935112; LONP, HSS113887)

19 (Thermo Fisher Scientific) or 5 nM Silencer select siRNAs (Silencer Select Negative Control \#1 siRNA,

20 4390843; OMA1, s41776; LONP, s17903; PTPMT1, s229947) (Thermo Fisher Scientific) were

21 transfected using Lipofectamine RNAi max transfection reagent (Thermo Fisher Scientific) at the same

22 time as cell seeding. For drug treatment experiments, cells were incubated in medium containing one or

23 more of the following compounds: CCCP (Cayman Chemical), MG132 (Sigma), Chlorhexidine (Cayman

24 Chemical), Alexidine (Cayman Chemical), Rotenone (Cayman Chemical), Actinonin (Cayman Chemical),

25 CDDO (Cayman Chemical). For examination of mitochondrial membrane potential or mitochondrial

26 ROS production, $20 \mathrm{nM}$ TMRM (Thermo Fisher Scientific) or $50 \mathrm{nM}$ MitoSOX (Thermo Fisher 
1 Scientific) respectively was directly added to cell culture media and incubated for 15 min. For NAO

2 staining, cells were washed twice with PBS and were incubated with 100 nM NAO (Thermo Fisher

3 Scientific) for $15 \mathrm{~min}$. Cells were washed and replaced with normal medium followed by live cell

4 imaging using a 63 × /1.4 NA oil immersion objective on Leica SP8 LIGHTNING Confocal Microscope

5 (Leica) or FACS analysis using Attune NxT Acoustic Focusing Cytometer (Thermo Fisher Scientific).

\section{$7 \quad$ Plasmids}

8 Site-directed mutagenesis of pLenti-CMV-Neo-PINK1 (C125G)-EYFP or pLVX-puro-OMA1 (E328Q)-

9 EYFP was performed by PCR amplification (CloneAmp HiFi PCR Premix, Takara or Q5 High-Fidelity

10 DNA Polymerase system, NEB) of PINK1 or OMA1 encoding plasmid using appropriate primers

11 followed by Gibson assembly (In-Fusion HD Cloning system, Clontech) into the SalI-XhoI of the pLenti-

12 CMV-Neo vector, or into the EcoRI site of pLVX-puro vector (Clontech). pLVX-puro-DELE1-HA and

13 pLVX-puro-Su9-mCherry was created by PCR amplification and sub-cloning into the EcoRI site of

14 pLVX-puro vector. All constructs were confirmed by DNA sequencing.

\section{Generation of stable cell lines}

17 To generate stably transfected cell lines, lentiviruses (for plasmids within pLenti-CMV-neo or pLVX-

18 puro vectors) were packaged in HEK293T cells. HeLa cells were transduced with viruses with $10 \mu \mathrm{g} / \mathrm{ml}$

19 polybrene (Sigma) then optimized for protein expression via antibiotics selection. PINK1 KO HeLa cells

20 stably expressed PINK1 (C125G)-EYFP were mono-cloned, and clone \#23 was used in this study.

\section{Immunoblotting (IB)}

23 For SDS-PAGE, cells were lysed with 1× NuPAGE LDS sample Buffer (Thermo Fisher Scientific)

24 supplemented with $100 \mathrm{mM}$ Dithiothreitol (DTT) (Sigma), and boiled with shaking for $15 \mathrm{~min}$.

25 Approximately, $50 \mu \mathrm{g}$ of protein per sample was separated on $7.5 \%, 10 \%$ or 4-20\% gradient Mini- 
1 PROTEAN TGX Precast Gel (Bio-Rad) or Criterion TGX Gels (Bio-Rad) and then transferred to a

2 nitrocellulose membrane (Bio-Rad) or PVDF membrane (Bio-Rad). The membrane was blocked with

3 Odyssey Blocking Buffer (LI-COR) and incubated with the indicated primary antibodies at $4^{\circ} \mathrm{C}$ overnight.

4 After washing with PBS-T (PBS + 0.05\% Tween-20), the membrane was incubated with HRP-conjugated

5 secondary antibodies (Thermo Fisher Scientific) and washed again with PBS-T. Detection was performed

6 with iBright CL1000 Imaging System (Thermo Fisher Scientific). For Phos-tag SDS-PAGE, cells were

7 lysed with $1 \%$ Triton buffer [1\% Triton-X100, $150 \mathrm{mM} \mathrm{NaCl}, 50 \mathrm{mM}$ Tris- $\mathrm{HCl} \mathrm{pH}$ 7.4, $1 \mathrm{mM}$ EDTA,

8 Phosphatase inhibitors (PhosSTOP, Sigma) and protease inhibitors (cOmplete, Sigma)]. After

9 centrifugation, the lysate that contains $10 \mu \mathrm{g}$ of protein per sample was mixed with $2 \mathrm{x}$ Laemmli Sample

10 Buffer (Bio-Rad) supplemented with 2 M 2-Mercaptoethanol (Bio-Rad), and boiled for 3 min. Samples

11 were separated on $7.5 \%$ Mini-Gel (TGX FastCast Acrylamide Solutions, Bio-Rad) containing $50 \mu \mathrm{M}$

12 Phos-tag AAL-107 (Wako) and $10 \mathrm{mM} \mathrm{MnCl}_{2}$ (Sigma) according to the manufacturer's instruction. For

13 the elimination of the manganese ion from the gel, the gel was soaked with a transfer buffer containing 1

14 mM EDTA for $10 \mathrm{~min}$, and washed with a transfer buffer without EDTA for $10 \mathrm{~min}$, and then transferred

15 to a PVDF membrane (Bio-Rad).

16

\section{Immunofluorescent chemistry (IF)}

18 Cells were seeded into Lab-Tek Chambered Coverglass with 4 wells (Thermo Fisher Scientific). Cells

19 were rinsed in PBS and fixed with PBS containing 4\% formaldehyde for $15 \mathrm{~min}$ at room temperature.

20 Cells were permeabilized with PBS containing $0.1 \%$ Triton X-100 for $10 \mathrm{~min}$ at room temperature.

21 Blocking was performed using PBS containing 2\% BSA for 30-60 min at room temperature. For

22 immunostaining, cells were incubated with primary or secondary antibodies (Alexa Fluor, Thermo Fisher

23 Scientific) diluted in PBS containing 2\% BSA for overnight at $4 \mathrm{C}^{\circ}$ or about 2 hours at room temperature,

24 respectively. Cells were imaged using a $63 \times / 1.4$ NA oil immersion objective on Leica SP8 LIGHTNING

25 Confocal Microscope (Leica). 


\section{Antibodies}

3 Following antibodies were used in IB or IF; PINK1 (Cell Signaling, 6946S), OMA1 (Santa Cruz, sc-

4 515788), PGAM5 (Thermo Fisher Scientific, PA5-57894), CHCHD2 (Proteintech, 66302-1-lg),

5 CHCHD10 (Sigma, HPA003440), OPA1 (BD Biosciences, 612607), HSP90 $\alpha / \beta$ (Santa Cruz, sc-7947 or

6 sc-13119), HSP60 (Santa Cruz, sc-13115), Tim50 (Santa Cruz, sc-393678), Tom20 (Santa Cruz, sc-

7 17764), PHB2 (Proteintech, 66424-1-1g), Cox IV (Thermo Fisher Scientific, PA5-19471), Mic60

8 (Proteintech, 10179-1-AP), HMOX1 (GeneTex, GTX101147), PTPMT1 (Santa Cruz, sc-390901),

9 COX17 (Proteintech, 11464-1-AP), COA7 (Proteintech, 25361-1-AP), LONP (Novus Biologicals, NBP1-

10 81734), GFP (Novus Biologicals, NB600-597 or NB-600-308), and HA (Cell Signaling, 3724).

\section{FDA-Approved Compound Library Screening}

13 The FDA-approved compound library (Selleck, $100 \mathrm{~nL}$ per drug) was stamped to black 384 well plates

14 with glass bottom using CyBio Well vario (Analytik Jena). PINK1 KO HeLa cells stably expressed 15 PINK1 (C125G)-EYFP (clone \#23) were then added to give density of 4000 cells per well and a final 16 drug concentration of $5 \mu \mathrm{M}$. After 18 hours of treatment, CCCP was added to a final concentration of 20 $17 \mu \mathrm{M}$ and incubated for 4 hours followed by fixation in $4 \%$ paraformaldehyde and counterstaining with 18 Hoechst 33342. Fluorescence was detected using an ImageXpress Micro XLS (Molecular devices) high19 content imager and cellular EYFP fluorescence signal was calculated using CellProfiler software [77].

\section{In vitro NAO assay}

22 Binding of NAO to each anionic phospholipid was studied in lipid monolayers [42, 43], with slight 23 modifications. 96 well microtiter plates (Corning, 3915) were coated with $50 \mu \mathrm{L}$ of $20 \mu \mathrm{M}$ each anionic 24 phospholipid in ethanol and evaporated at $37^{\circ} \mathrm{C}$ for 5 hours. Increasing concentration of Alexidine (final; $250-100 \mu \mathrm{M}$ ) or CCCP (final; $10 \mu \mathrm{M}$ ) in $50 \mu \mathrm{L}$ PBS containing $2 \%$ BSA was added and incubated at $37^{\circ} \mathrm{C}$ 26 for 30 min. Then, NAO (final; $100 \mathrm{nM}$ ) in $50 \mu \mathrm{L}$ PBS containing $2 \%$ BSA was added and incubated at 
$137^{\circ} \mathrm{C}$ for $30 \mathrm{~min}$ protected from light. After the incubation, each well of the plates was washed with 150

$2 \mu \mathrm{L}$ PBS for 5 times. Finally, NAO fluorescence intensity was measured by SpectraMax i3x Multi-Mode

3 Detection Platform (Molecular Devices) using Ex 485nm /Em 535 nm. Following anionic phospholipids

4 purchased from Avanti Polar Lipids were used; Heart CA (840012P), Egg PA (840101P), Egg PG

5 (841138P), Liver PI (840042P), and Brain PS (840032P).

\section{Transmission Electron Microscopy}

8 HeLa cells were fixed with $4 \%$ glutaraldehyde (Electron Microscopy Services) in EM buffer $(0.1 \mathrm{~N}$

9 sodium cacodylate at $\mathrm{pH} 7.4$ with $2 \mathrm{mM}$ calcium chloride) for $30 \mathrm{~min}$ at room temperature and then at

$10 \quad 4^{\circ} \mathrm{C}$ for at least 24 hours. Samples were washed with buffer and treated with $1 \%$ osmium tetroxide in 0.1

$11 \mathrm{~N}$ cacodylate buffer at $\mathrm{pH} 7.4$ for 1 hour on ice, washed and en bloc stained with $0.25-1 \%$ uranyl acetate

12 in $0.1 \mathrm{~N}$ acetate buffer at $\mathrm{pH} 5.0$ overnight at $4{ }^{\circ} \mathrm{C}$, dehydrated with a series of graded ethanol and finally

13 embedded in epoxy resins. Ultrathin sections $(70 \mathrm{~nm})$ were stained with lead citrate and imaged with a

14 JEOL 1200 EXII Transmission Electron Microscope.

\section{RNA Isolation and Real-Time PCR}

17 Total RNAs were isolated using RNeasy Mini Kit (Qiagen) and reverse-transcribed to cDNA using 18 iScript cDNA Synthesis Kit (Bio-Rad) according to the manufacturer's instruction. Real-Time (RT) PCR 19 was performed using SYBR Green Master Mix (Bio-Rad) and QuantStudio 3 RT-PCR system (Thermo

20 Fisher Scientific). All expression levels were normalized to that of RPS18 mRNA. The following RT-

21 PCR primers were used; RPS18, (forward) 5'- cttccacaggaggcctacac-3' and (reverse) 5'-

22 cgcaaaatatgctggaacttt-3'; HMXO1, (forward) 5'- ggcagagggtgatagaagagg-3' and (reverse) 5'-

23 agctcctgcaactcctcaaa-3'; MT2A, (forward) 5'- aacctgtcccgactctagcc-3' and (reverse) 5'-

24 Gcaggtgcaggagtcacc-3'; COA7, (forward) 5'-gcaggtcaagtcctttttgg-3' and (reverse) 5'-

25 ccaccagccgatagcaac-3'; COX17, (forward) 5'-aagatgccgggtctggtt-3' and (reverse) 5' 
ttcttctccttctcgatgataca-3'; mtHSP60, (forward) 5'-cctgcactctgtccetcact-3' and (reverse) 5' -

2 gggtaaccgaagcatttctg-3'.

4 TMT-based quantitative proteomics

5 Sample Preparation - Cells were lysed in $8 \mathrm{M}$ urea, $50 \mathrm{mM}$ Tris- $\mathrm{HCl}$ ( $\mathrm{pH} 8.0), 1 \mathrm{X}$ Complete Protease

6 Inhibitor (Roche) and 1X PhosStop (Roche) with a sonic probe (3 x 30s at $80 \%$ amplitude) with

7 subsequent mixing at room temperature for 1 hour at $1000 \mathrm{rpm}$ on a Thermomixer. Lysates were

8 quantified by Qubit fluorometry (Life Technologies). $50 \mu \mathrm{g}$ of each sample was digested overnight with

9 trypsin. Briefly, samples were reduced for 1 hour at room temperature in $12 \mathrm{mM}$ DTT followed by

10 alkylation for 1 hour at room temperature in $15 \mathrm{mM}$ iodoacetamide. Trypsin was added to an

11 enzyme:substrate ratio of 1:20. Each sample was acidified in formic acid and subjected to SPE on an

12 Empore SD C18 plate. Each sample was lyophilized and reconstituted in $140 \mathrm{mM}$ HEPES (pH 8.0), 30\%

13 acetonitrile for TMT labeling. Peptides were labeled using TMT 10-plex (Thermo Fisher Scientific)

14 according to manufacturer's instructions. Briefly, $40 \mu \mathrm{L}$ of acetonitrile was added to each TMT tag tube

15 and mixed aggressively. Tags were incubated at room temperature for $15 \mathrm{~min} .30 \mu \mathrm{L}$ of label was added

16 to each peptide sample and mixed aggressively. Samples were incubated in an Eppendorf Thermomixer at

$17300 \mathrm{rpm}$ at $25^{\circ} \mathrm{C}$ for 1.5 hour. Reactions were terminated with the addition of $8 \mu \mathrm{L}$ of fresh $5 \%$

18 hydroxylamine solution and $15 \mathrm{~min}$ incubation at room temperature. Each labeled sample was pooled,

19 frozen, and lyophilized and subjected to SPE on a High-Density 3M Empore SDB-XC column. The

20 eluent was lyophilized. Peptides were fractionated using high $\mathrm{pH}$ reverse-phase chromatography on an

21 Agilent 1100 HPLC system using a Waters XBridge C18 column (2.1mm ID x 150mm length, $3.5 \mu \mathrm{m}$

22 particle size) at $300 \mu \mathrm{L} / \mathrm{min}$. The following gradient was employed: 0.5\% B initial conditions, $0.5-3.0 \% \mathrm{~B}$

23 from 0-1 min, 3-25\% B from 1-36 min, 25\%-45\% B from 36-44 min, 45-90\% B from 44-47 min, 90\% B

24 from 47-49 min, 90\%-0.5\% buffer B from 49-50 min (buffer A: 100\% $\mathrm{H}_{2} \mathrm{O}, 10 \mathrm{mM} \mathrm{NH} \mathrm{OH}_{4}$; buffer B:

$\left.25100 \% \mathrm{CH}_{3} \mathrm{CN}, 10 \mathrm{mM} \mathrm{NH} \mathrm{NH}_{4} \mathrm{OH}\right)$. Every $12^{\text {th }}$ well was combined to create 12 pools. Each pool was

26 lyophilized. Mass Spectrometry - Peptides (10\% per pool) were analyzed by nano LC/MS/MS with a 
1 Waters NanoAcquity HPLC system interfaced to a ThermoFisher Fusion Lumos mass spectrometer.

2 Peptides were loaded on a trapping column and eluted over a $75 \mu \mathrm{m}$ analytical column at $350 \mathrm{~nL} / \mathrm{min}$;

3 both columns were packed with Luna C18 resin (Phenomenex). Each high pH RP pool was separated

4 over a 2 hours gradient (24 hours instrument time total). The mass spectrometer was operated in data-

5 dependent mode, with MS and MS/MS performed in the Orbitrap at 60,000 FWHM resolution and 50,000

6 FWHM resolution, respectively. A 3 s cycle time was employed for all steps. Data Analysis - Data were

$7 \quad$ analyzed using MaxQuant v1.6.2.3 (Max Planck) and searched against the combined forward and reverse

8 Swissprot $H$. sapiens protein database. The database was appended with common background proteins.

9 Search parameters were precursor mass tolerance $7 \mathrm{ppm}$, product ion mass tolerance $20 \mathrm{ppm}, 2$ missed

10 cleavages allowed, fully tryptic peptides only, fixed modification of carbamidomethyl cysteine, variable

11 modifications of oxidized methionine and protein N-terminal acetylation. Data were filtered $1 \%$ protein

12 and peptide level false discovery rate (FDR) and requiring at least one unique peptide per protein.

13 Reporter ion intensities were exported for further analysis.

\section{Statistical analysis}

16 Statistical significances were determined using Prism software (GraphPad Software, Inc.) as indicated in 17 the Figure legends.

\section{Acknowledgements}

20 We thank Dr. Toren Finkel for critical reading our manuscript and thank Dr. Richard J Youle for sharing 21 materials. We thank Dr. Susan Cheng, Ms. Sandra Lara, and the NINDS, EM Facility (NIH) for technical 22 assistance with transmission electron microscopy. We thank Dr. Richard Jones and MS Bioworks, LLC

23 for performing TMT-based quantitative proteomics. This work was supported by University of Pittsburgh,

24 Aging Institute Startup Seed, Samuel and Emma Winters Foundation, and UPMC Health System

25 Competitive Medical Research Fund. This work was supported (in part) by the NINDS intramural 26 program. 


\section{Author contributions}

3 R.H., Y.S., and S.S. designed and performed experiments and wrote the manuscript. M.L. and B.B.C.

4 performed the FDA-approved compound screening. K.M. provided chemical-structural insights on hit 5 compounds. D.P.N. designed the EM analysis and interpreted the EM images.

7 Conflict of interest

8 The authors declare no competing financial interest.

\section{References}

16 1. Horvath SE, Daum G. Lipids of mitochondria. Prog Lipid Res. 2013;52(4):590-614. Epub

17 2013/09/07. doi: 10.1016/j.plipres.2013.07.002. PubMed PMID: 24007978.

18 2. Tamura Y, Kawano S, Endo T. Lipid homeostasis in mitochondria. Biol Chem. 2020;401(6-

19 7):821-33. Epub 2020/04/02. doi: 10.1515/hsz-2020-0121. PubMed PMID: 32229651.

20 3. Tatsuta T, Langer T. Intramitochondrial phospholipid trafficking. Biochim Biophys Acta Mol

21 Cell Biol Lipids. 2017;1862(1):81-9. Epub 2016/08/21. doi: 10.1016/j.bbalip.2016.08.006. PubMed 22 PMID: 27542541.

23 4. Cogliati S, Enriquez JA, Scorrano L. Mitochondrial Cristae: Where Beauty Meets Functionality.

24 Trends Biochem Sci. 2016;41(3):261-73. Epub 2016/02/10. doi: 10.1016/j.tibs.2016.01.001. PubMed 25 PMID: 26857402. 
15 5oule RJ. Mitochondria-Striking a balance between host and endosymbiont. Science.

2 2019;365(6454). Epub 2019/08/17. doi: 10.1126/science.aaw9855. PubMed PMID: 31416937.

3 6. Youle RJ, Narendra DP. Mechanisms of mitophagy. Nat Rev Mol Cell Biol. 2011;12(1):9-14.

4 Epub 2010/12/24. doi: 10.1038/nrm3028. PubMed PMID: 21179058; PubMed Central PMCID:

$5 \quad$ PMCPMC4780047.

67 Anderson NS, Haynes CM. Folding the Mitochondrial UPR into the Integrated Stress Response.

7 Trends Cell Biol. 2020;30(6):428-39. Epub 2020/05/16. doi: 10.1016/j.tcb.2020.03.001. PubMed PMID:

8 32413314; PubMed Central PMCID: PMCPMC7230072.

9 8. Narendra D, Tanaka A, Suen DF, Youle RJ. Parkin is recruited selectively to impaired

10 mitochondria and promotes their autophagy. J Cell Biol. 2008;183(5):795-803. Epub 2008/11/26. doi:

11 10.1083/jcb.200809125. PubMed PMID: 19029340; PubMed Central PMCID: PMCPMC2592826.

12 9. Matsuda N, Sato S, Shiba K, Okatsu K, Saisho K, Gautier CA, et al. PINK1 stabilized by

13 mitochondrial depolarization recruits Parkin to damaged mitochondria and activates latent Parkin for

14 mitophagy. J Cell Biol. 2010;189(2):211-21. Epub 2010/04/21. doi: 10.1083/jcb.200910140. PubMed

15 PMID: 20404107; PubMed Central PMCID: PMCPMC2856912.

16 10. Lazarou M, Sliter DA, Kane LA, Sarraf SA, Wang C, Burman JL, et al. The ubiquitin kinase

17 PINK1 recruits autophagy receptors to induce mitophagy. Nature. 2015;524(7565):309-14. Epub

18 2015/08/13. doi: 10.1038/nature14893. PubMed PMID: 26266977; PubMed Central PMCID:

19 PMCPMC5018156.

20 11. Munch C, Harper JW. Mitochondrial unfolded protein response controls matrix pre-RNA

21 processing and translation. Nature. 2016;534(7609):710-3. Epub 2016/06/29. doi: 10.1038/nature18302.

22 PubMed PMID: 27350246; PubMed Central PMCID: PMCPMC4939261.

23 12. Burman JL, Pickles S, Wang C, Sekine S, Vargas JNS, Zhang Z, et al. Mitochondrial fission

24 facilitates the selective mitophagy of protein aggregates. J Cell Biol. 2017;216(10):3231-47. Epub

25 2017/09/13. doi: 10.1083/jcb.201612106. PubMed PMID: 28893839; PubMed Central PMCID:

26 PMCPMC5626535. 
1 13. Richter U, Lahtinen T, Marttinen P, Suomi F, Battersby BJ. Quality control of mitochondrial

2 protein synthesis is required for membrane integrity and cell fitness. J Cell Biol. 2015;211(2):373-89.

3 Epub 2015/10/28. doi: 10.1083/jcb.201504062. PubMed PMID: 26504172; PubMed Central PMCID:

4 PMCPMC4621829.

5 14. Hasson SA, Damoiseaux R, Glavin JD, Dabir DV, Walker SS, Koehler CM. Substrate specificity

6 of the TIM22 mitochondrial import pathway revealed with small molecule inhibitor of protein

7 translocation. Proc Natl Acad Sci U S A. 2010;107(21):9578-83. Epub 2010/05/12. doi:

8 10.1073/pnas.0914387107. PubMed PMID: 20457929; PubMed Central PMCID: PMCPMC2906835.

9 15. Dabir DV, Hasson SA, Setoguchi K, Johnson ME, Wongkongkathep P, Douglas CJ, et al. A 10 small molecule inhibitor of redox-regulated protein translocation into mitochondria. Dev Cell. 11 2013;25(1):81-92. Epub 2013/04/20. doi: 10.1016/j.devcel.2013.03.006. PubMed PMID: 23597483;

12 PubMed Central PMCID: PMCPMC3726224.

13 16. Quiros PM, Prado MA, Zamboni N, D'Amico D, Williams RW, Finley D, et al. Multi-omics 14 analysis identifies ATF4 as a key regulator of the mitochondrial stress response in mammals. J Cell Biol. 15 2017;216(7):2027-45. Epub 2017/06/02. doi: 10.1083/jcb.201702058. PubMed PMID: 28566324; 16 PubMed Central PMCID: PMCPMC5496626.

17 17. Fessler E, Eckl EM, Schmitt S, Mancilla IA, Meyer-Bender MF, Hanf M, et al. A pathway 18 coordinated by DELE1 relays mitochondrial stress to the cytosol. Nature. 2020;579(7799):433-7. Epub 19 2020/03/07. doi: 10.1038/s41586-020-2076-4. PubMed PMID: 32132706.

20 18. Guo X, Aviles G, Liu Y, Tian R, Unger BA, Lin YT, et al. Mitochondrial stress is relayed to the 21 cytosol by an OMA1-DELE1-HRI pathway. Nature. 2020;579(7799):427-32. Epub 2020/03/07. doi: 22 10.1038/s41586-020-2078-2. PubMed PMID: 32132707; PubMed Central PMCID: PMCPMC7147832.

23 19. Jin SM, Lazarou M, Wang C, Kane LA, Narendra DP, Youle RJ. Mitochondrial membrane 24 potential regulates PINK1 import and proteolytic destabilization by PARL. J Cell Biol. 2010;191(5):93325 42. Epub 2010/12/01. doi: 10.1083/jcb.201008084. PubMed PMID: 21115803; PubMed Central PMCID: 26 PMCPMC2995166. 
1 20. Yamano K, Youle RJ. PINK1 is degraded through the N-end rule pathway. Autophagy.

2 2013;9(11):1758-69. Epub 2013/10/15. doi: 10.4161/auto.24633. PubMed PMID: 24121706; PubMed

3 Central PMCID: PMCPMC4028335.

4 21. Narendra DP, Jin SM, Tanaka A, Suen DF, Gautier CA, Shen J, et al. PINK1 is selectively

5 stabilized on impaired mitochondria to activate Parkin. PLoS Biol. 2010;8(1):e1000298. Epub 2010/02/04.

6 doi: 10.1371/journal.pbio.1000298. PubMed PMID: 20126261; PubMed Central PMCID:

$7 \quad$ PMCPMC2811155.

8 22. Okatsu K, Oka T, Iguchi M, Imamura K, Kosako H, Tani N, et al. PINK1 autophosphorylation

9 upon membrane potential dissipation is essential for Parkin recruitment to damaged mitochondria. Nat

10 Commun. 2012;3:1016. Epub 2012/08/23. doi: 10.1038/ncomms2016. PubMed PMID: 22910362;

11 PubMed Central PMCID: PMCPMC3432468.

12 23. Sekine S, Wang C, Sideris DP, Bunker E, Zhang Z, Youle RJ. Reciprocal Roles of Tom7 and

13 OMA1 during Mitochondrial Import and Activation of PINK1. Mol Cell. 2019;73(5):1028-43 e5. Epub

14 2019/02/09. doi: 10.1016/j.molcel.2019.01.002. PubMed PMID: 30733118.

15 24. Sekine S. PINK1 import regulation at a crossroad of mitochondrial fate: the molecular 16 mechanisms of PINK1 import. J Biochem. 2020;167(3):217-24. Epub 2019/09/11. doi:

17 10.1093/jb/mvz069. PubMed PMID: 31504668.

18 25. Zhang JH, Chung TD, Oldenburg KR. A Simple Statistical Parameter for Use in Evaluation and

19 Validation of High Throughput Screening Assays. J Biomol Screen. 1999;4(2):67-73. Epub 2000/06/06.

20 doi: 10.1177/108705719900400206. PubMed PMID: 10838414.

21 26. McDonnell G, Russell AD. Antiseptics and disinfectants: activity, action, and resistance. Clin

22 Microbiol Rev. 1999;12(1):147-79. Epub 1999/01/09. PubMed PMID: 9880479; PubMed Central

23 PMCID: PMCPMC88911.

24 27. Cieplik F, Jakubovics NS, Buchalla W, Maisch T, Hellwig E, Al-Ahmad A. Resistance Toward 25 Chlorhexidine in Oral Bacteria - Is There Cause for Concern? Front Microbiol. 2019;10:587. Epub 
1 2019/04/11. doi: 10.3389/fmicb.2019.00587. PubMed PMID: 30967854; PubMed Central PMCID:

2 PMCPMC6439480.

3 28. Ehses S, Raschke I, Mancuso G, Bernacchia A, Geimer S, Tondera D, et al. Regulation of OPA1

4 processing and mitochondrial fusion by m-AAA protease isoenzymes and OMA1. J Cell Biol.

5 2009;187(7):1023-36. Epub 2009/12/30. doi: 10.1083/jcb.200906084. PubMed PMID: 20038678;

6 PubMed Central PMCID: PMCPMC2806285.

7 29. Head B, Griparic L, Amiri M, Gandre-Babbe S, van der Bliek AM. Inducible proteolytic

8 inactivation of OPA1 mediated by the OMA1 protease in mammalian cells. J Cell Biol. 2009;187(7):959-

9 66. Epub 2009/12/30. doi: 10.1083/jcb.200906083. PubMed PMID: 20038677; PubMed Central PMCID:

10 PMCPMC2806274.

11 30. Baker MJ, Lampe PA, Stojanovski D, Korwitz A, Anand R, Tatsuta T, et al. Stress-induced

12 OMA1 activation and autocatalytic turnover regulate OPA1-dependent mitochondrial dynamics. EMBO J.

13 2014;33(6):578-93. Epub 2014/02/20. doi: 10.1002/embj.201386474. PubMed PMID: 24550258;

14 PubMed Central PMCID: PMCPMC3989652.

15 31. Zhang K, Li H, Song Z. Membrane depolarization activates the mitochondrial protease OMA1 by 16 stimulating self-cleavage. EMBO Rep. 2014;15(5):576-85. Epub 2014/04/11. doi:

17 10.1002/embr.201338240. PubMed PMID: 24719224; PubMed Central PMCID: PMCPMC4210089.

18 32. Sekine S, Kanamaru Y, Koike M, Nishihara A, Okada M, Kinoshita H, et al. Rhomboid protease

19 PARL mediates the mitochondrial membrane potential loss-induced cleavage of PGAM5. J Biol Chem.

20 2012;287(41):34635-45. Epub 2012/08/24. doi: 10.1074/jbc.M112.357509. PubMed PMID: 22915595;

21 PubMed Central PMCID: PMCPMC3464569.

22 33. Wai T, Saita S, Nolte H, Muller S, Konig T, Richter-Dennerlein R, et al. The membrane scaffold

23 SLP2 anchors a proteolytic hub in mitochondria containing PARL and the i-AAA protease YME1L.

24 EMBO Rep. 2016;17(12):1844-56. Epub 2016/10/16. doi: 10.15252/embr.201642698. PubMed PMID:

25 27737933; PubMed Central PMCID: PMCPMC5283581. 
1 34. Liu YT, Huang X, Nguyen D, Shammas MK, Wu BP, Dombi E, et al. Loss of CHCHD2 and

2 CHCHD10 activates OMA1 peptidase to disrupt mitochondrial cristae phenocopying patient mutations.

3 Hum Mol Genet. 2020;29(9):1547-67. Epub 2020/04/28. doi: 10.1093/hmg/ddaa077. PubMed PMID:

4 32338760; PubMed Central PMCID: PMCPMC7268789.

5 35. Doughty-Shenton D, Joseph JD, Zhang J, Pagliarini DJ, Kim Y, Lu D, et al. Pharmacological

6 targeting of the mitochondrial phosphatase PTPMT1. J Pharmacol Exp Ther. 2010;333(2):584-92. Epub

7 2010/02/20. doi: 10.1124/jpet.109.163329. PubMed PMID: 20167843; PubMed Central PMCID:

$8 \quad$ PMCPMC2872949.

9 36. Zhang J, Guan Z, Murphy AN, Wiley SE, Perkins GA, Worby CA, et al. Mitochondrial

10 phosphatase PTPMT1 is essential for cardiolipin biosynthesis. Cell Metab. 2011;13(6):690-700. Epub

11 2011/06/07. doi: 10.1016/j.cmet.2011.04.007. PubMed PMID: 21641550; PubMed Central PMCID:

12 PMCPMC3119201.

13 37. Xiao J, Engel JL, Zhang J, Chen MJ, Manning G, Dixon JE. Structural and functional analysis of

14 PTPMT1, a phosphatase required for cardiolipin synthesis. Proc Natl Acad Sci U S A.

15 2011;108(29):11860-5. Epub 2011/07/07. doi: 10.1073/pnas.1109290108. PubMed PMID: 21730175;

16 PubMed Central PMCID: PMCPMC3142007.

17 38. Langmaier J, Pizl M, Samec Z, Zalis S. Extreme Basicity of Biguanide Drugs in Aqueous

18 Solutions: Ion Transfer Voltammetry and DFT Calculations. J Phys Chem A. 2016;120(37):7344-50.

19 Epub 2016/08/27. doi: 10.1021/acs.jpca.6b04786. PubMed PMID: 27564915.

20 39. Wolf DM, Segawa M, Kondadi AK, Anand R, Bailey ST, Reichert AS, et al. Individual cristae

21 within the same mitochondrion display different membrane potentials and are functionally independent.

22 EMBO J. 2019;38(22):e101056. Epub 2019/10/15. doi: 10.15252/embj.2018101056. PubMed PMID:

23 31609012; PubMed Central PMCID: PMCPMC6856616.

24 40. Kondadi AK, Anand R, Hansch S, Urbach J, Zobel T, Wolf DM, et al. Cristae undergo 25 continuous cycles of membrane remodelling in a MICOS-dependent manner. EMBO Rep. 
1 2020;21(3):e49776. Epub 2020/02/19. doi: 10.15252/embr.201949776. PubMed PMID: 32067344;

2 PubMed Central PMCID: PMCPMC7054676.

3 41. Lin TY, Weibel DB. Organization and function of anionic phospholipids in bacteria. Appl

4 Microbiol Biotechnol. 2016;100(10):4255-67. Epub 2016/03/31. doi: 10.1007/s00253-016-7468-x.

$5 \quad$ PubMed PMID: 27026177.

6 42. Rodriguez ME, Azizuddin K, Zhang P, Chiu SM, Lam M, Kenney ME, et al. Targeting of

7 mitochondria by $10-\mathrm{N}$-alkyl acridine orange analogues: role of alkyl chain length in determining cellular

8 uptake and localization. Mitochondrion. 2008;8(3):237-46. Epub 2008/06/03. doi:

9 10.1016/j.mito.2008.04.003. PubMed PMID: 18514589; PubMed Central PMCID: PMCPMC2585370.

10 43. Nomura K, Imai H, Koumura T, Kobayashi T, Nakagawa Y. Mitochondrial phospholipid

11 hydroperoxide glutathione peroxidase inhibits the release of cytochrome $\mathrm{c}$ from mitochondria by

12 suppressing the peroxidation of cardiolipin in hypoglycaemia-induced apoptosis. Biochem J. 2000;351(Pt

13 1):183-93. Epub 2000/09/22. doi: 10.1042/0264-6021:3510183. PubMed PMID: 10998361; PubMed

14 Central PMCID: PMCPMC1221349.

15 44. Battogtokh G, Choi YS, Kang DS, Park SJ, Shim MS, Huh KM, et al. Mitochondria-targeting

16 drug conjugates for cytotoxic, anti-oxidizing and sensing purposes: current strategies and future

17 perspectives. Acta Pharm Sin B. 2018;8(6):862-80. Epub 2018/12/07. doi: 10.1016/j.apsb.2018.05.006.

18 PubMed PMID: 30505656; PubMed Central PMCID: PMCPMC6251809.

19 45. Sibrian-Vazquez M, Nesterova IV, Jensen TJ, Vicente MG. Mitochondria targeting by guanidine-

20 and biguanidine-porphyrin photosensitizers. Bioconjug Chem. 2008;19(3):705-13. Epub 2008/02/14. doi:

21 10.1021/bc700393u. PubMed PMID: 18269224.

22 46. Rampelt H, Zerbes RM, van der Laan M, Pfanner N. Role of the mitochondrial contact site and

23 cristae organizing system in membrane architecture and dynamics. Biochim Biophys Acta Mol Cell Res.

24 2017;1864(4):737-46. Epub 2016/09/11. doi: 10.1016/j.bbamcr.2016.05.020. PubMed PMID: 27614134. 
1 47. Tatsuta T, Model K, Langer T. Formation of membrane-bound ring complexes by prohibitins in

2 mitochondria. Mol Biol Cell. 2005;16(1):248-59. Epub 2004/11/05. doi: 10.1091/mbc.e04-09-0807.

3 PubMed PMID: 15525670; PubMed Central PMCID: PMCPMC539169.

4 48. Osman C, Merkwirth C, Langer T. Prohibitins and the functional compartmentalization of

5 mitochondrial membranes. J Cell Sci. 2009;122(Pt 21):3823-30. Epub 2009/11/06. doi:

6 10.1242/jcs.037655. PubMed PMID: 19889967.

7 49. Richter-Dennerlein R, Korwitz A, Haag M, Tatsuta T, Dargazanli S, Baker M, et al. DNAJC19, a

8 mitochondrial cochaperone associated with cardiomyopathy, forms a complex with prohibitins to regulate

9 cardiolipin remodeling. Cell Metab. 2014;20(1):158-71. Epub 2014/05/27. doi:

10 10.1016/j.cmet.2014.04.016. PubMed PMID: 24856930.

11 50. Osman C, Haag M, Potting C, Rodenfels J, Dip PV, Wieland FT, et al. The genetic interactome of

12 prohibitins: coordinated control of cardiolipin and phosphatidylethanolamine by conserved regulators in

13 mitochondria. J Cell Biol. 2009;184(4):583-96. Epub 2009/02/18. doi: 10.1083/jcb.200810189. PubMed

14 PMID: 19221197; PubMed Central PMCID: PMCPMC2654118.

15 51. Steglich G, Neupert W, Langer T. Prohibitins regulate membrane protein degradation by the m-

16 AAA protease in mitochondria. Mol Cell Biol. 1999;19(5):3435-42. Epub 1999/04/17. doi:

17 10.1128/mcb.19.5.3435. PubMed PMID: 10207067; PubMed Central PMCID: PMCPMC84136.

18 52. Merkwirth C, Martinelli P, Korwitz A, Morbin M, Bronneke HS, Jordan SD, et al. Loss of

19 prohibitin membrane scaffolds impairs mitochondrial architecture and leads to tau hyperphosphorylation

20 and neurodegeneration. PLoS Genet. 2012;8(11):e1003021. Epub 2012/11/13. doi:

21 10.1371/journal.pgen.1003021. PubMed PMID: 23144624; PubMed Central PMCID: PMCPMC3493444.

22 53. Korwitz A, Merkwirth C, Richter-Dennerlein R, Troder SE, Sprenger HG, Quiros PM, et al. Loss

23 of OMA1 delays neurodegeneration by preventing stress-induced OPA1 processing in mitochondria. J

24 Cell Biol. 2016;212(2):157-66. Epub 2016/01/20. doi: 10.1083/jcb.201507022. PubMed PMID: $2526783299 ;$ PubMed Central PMCID: PMCPMC4738383. 
1 54. Yan C, Gong L, Chen L, Xu M, Abou-Hamdan H, Tang M, et al. PHB2 (prohibitin 2) promotes

2 PINK1-PRKN/Parkin-dependent mitophagy by the PARL-PGAM5-PINK1 axis. Autophagy.

3 2020;16(3):419-34. Epub 2019/06/11. doi: 10.1080/15548627.2019.1628520. PubMed PMID: 31177901;

4 PubMed Central PMCID: PMCPMC6999623.

5 55. Hoppins S, Collins SR, Cassidy-Stone A, Hummel E, Devay RM, Lackner LL, et al. A

6 mitochondrial-focused genetic interaction map reveals a scaffold-like complex required for inner

7 membrane organization in mitochondria. J Cell Biol. 2011;195(2):323-40. Epub 2011/10/12. doi:

8 10.1083/jcb.201107053. PubMed PMID: 21987634; PubMed Central PMCID: PMCPMC3198156.

9 56. Friedman JR, Mourier A, Yamada J, McCaffery JM, Nunnari J. MICOS coordinates with

10 respiratory complexes and lipids to establish mitochondrial inner membrane architecture. Elife. 2015;4.

11 Epub 2015/04/29. doi: 10.7554/eLife.07739. PubMed PMID: 25918844; PubMed Central PMCID:

12 PMCPMC4434539.

13 57. Michaud M, Gros V, Tardif M, Brugiere S, Ferro M, Prinz WA, et al. AtMic60 Is Involved in

14 Plant Mitochondria Lipid Trafficking and Is Part of a Large Complex. Curr Biol. 2016;26(5):627-39.

15 Epub 2016/02/24. doi: 10.1016/j.cub.2016.01.011. PubMed PMID: 26898467; PubMed Central PMCID:

16 PMCPMC6322921.

17 58. Weber TA, Koob S, Heide H, Wittig I, Head B, van der Bliek A, et al. APOOL is a cardiolipin-

18 binding constituent of the Mitofilin/MINOS protein complex determining cristae morphology in

19 mammalian mitochondria. PLoS One. 2013;8(5):e63683. Epub 2013/05/25. doi:

20 10.1371/journal.pone.0063683. PubMed PMID: 23704930; PubMed Central PMCID: PMCPMC3660581.

21 59. Igarashi K, Sun J. The heme-Bach1 pathway in the regulation of oxidative stress response and

22 erythroid differentiation. Antioxid Redox Signal. 2006;8(1-2):107-18. Epub 2006/02/21. doi:

23 10.1089/ars.2006.8.107. PubMed PMID: 16487043.

24 60. Kenny HA, Lal-Nag M, White EA, Shen M, Chiang CY, Mitra AK, et al. Quantitative high 25 throughput screening using a primary human three-dimensional organotypic culture predicts in vivo 
1 efficacy. Nat Commun. 2015;6:6220. Epub 2015/02/06. doi: 10.1038/ncomms7220. PubMed PMID:

2 25653139; PubMed Central PMCID: PMCPMC4427252.

3 61. Commander R, Wei C, Sharma A, Mouw JK, Burton LJ, Summerbell E, et al. Subpopulation

4 targeting of pyruvate dehydrogenase and GLUT1 decouples metabolic heterogeneity during collective

5 cancer cell invasion. Nat Commun. 2020;11(1):1533. Epub 2020/03/27. doi: 10.1038/s41467-020-15219-

6 7. PubMed PMID: 32210228; PubMed Central PMCID: PMCPMC7093428.

7 62. Liu X, Zheng H, Yu WM, Cooper TM, Bunting KD, Qu CK. Maintenance of mouse

8 hematopoietic stem cells ex vivo by reprogramming cellular metabolism. Blood. 2015;125(10):1562-5.

9 Epub 2015/01/17. doi: 10.1182/blood-2014-04-568949. PubMed PMID: 25593337; PubMed Central

10 PMCID: PMCPMC4351504.

11 63. Nath AK, Ryu JH, Jin YN, Roberts LD, Dejam A, Gerszten RE, et al. PTPMT1 Inhibition

12 Lowers Glucose through Succinate Dehydrogenase Phosphorylation. Cell Rep. 2015;10(5):694-701. Epub

13 2015/02/11. doi: 10.1016/j.celrep.2015.01.010. PubMed PMID: 25660020; PubMed Central PMCID:

14 PMCPMC4524786.

15 64. Wang C, Niederstrasser H, Douglas PM, Lin R, Jaramillo J, Li Y, et al. Small-molecule TFEB

16 pathway agonists that ameliorate metabolic syndrome in mice and extend C. elegans lifespan. Nat

17 Commun. 2017;8(1):2270. Epub 2017/12/24. doi: 10.1038/s41467-017-02332-3. PubMed PMID:

18 29273768; PubMed Central PMCID: PMCPMC5741634.

19 65. Smith A. Links between cell-surface events involving redox-active copper and gene regulation in

20 the hemopexin heme transport system. Antioxid Redox Signal. 2000;2(2):157-75. Epub 2001/03/07. doi:

21 10.1089/ars.2000.2.2-157. PubMed PMID: 11229523.

22 66. $\mathrm{Xu} \mathrm{W}$, Barrientos $\mathrm{T}$, Andrews NC. Iron and copper in mitochondrial diseases. Cell Metab.

23 2013;17(3):319-28. Epub 2013/03/12. doi: 10.1016/j.cmet.2013.02.004. PubMed PMID: 23473029;

24 PubMed Central PMCID: PMCPMC3594794.

25 67. Mohanraj K, Wasilewski M, Beninca C, Cysewski D, Poznanski J, Sakowska P, et al. Inhibition 26 of proteasome rescues a pathogenic variant of respiratory chain assembly factor COA7. EMBO Mol Med. 
1 2019;11(5). Epub 2019/03/20. doi: 10.15252/emmm.201809561. PubMed PMID: 30885959; PubMed

2 Central PMCID: PMCPMC6505684.

3 68. Kozjak-Pavlovic V, Prell F, Thiede B, Gotz M, Wosiek D, Ott C, et al. C1orf163/RESA1 is a

4 novel mitochondrial intermembrane space protein connected to respiratory chain assembly. J Mol Biol.

5 2014;426(4):908-20. Epub 2013/12/18. doi: 10.1016/j.jmb.2013.12.001. PubMed PMID: 24333015.

6 69. Merkwirth C, Dargazanli S, Tatsuta T, Geimer S, Lower B, Wunderlich FT, et al. Prohibitins

7 control cell proliferation and apoptosis by regulating OPA1-dependent cristae morphogenesis in

8 mitochondria. Genes Dev. 2008;22(4):476-88. Epub 2008/02/19. doi: 10.1101/gad.460708. PubMed

9 PMID: 18281461; PubMed Central PMCID: PMCPMC2238669.

10 70. Stephan T, Bruser C, Deckers M, Steyer AM, Balzarotti F, Barbot M, et al. MICOS assembly

11 controls mitochondrial inner membrane remodeling and crista junction redistribution to mediate cristae

12 formation. EMBO J. 2020;39(14):e104105. Epub 2020/06/23. doi: 10.15252/embj.2019104105. PubMed

13 PMID: 32567732; PubMed Central PMCID: PMCPMC7361284.

14 71. Tirrell PS, Nguyen KN, Luby-Phelps K, Friedman JR. MICOS subcomplexes assemble

15 independently on the mitochondrial inner membrane in proximity to ER contact sites. J Cell Biol.

16 2020;219(11). Epub 2020/10/15. doi: 10.1083/jcb.202003024. PubMed PMID: 33053165; PubMed

17 Central PMCID: PMCPMC7545361.

18 72. van der Laan M, Bohnert M, Wiedemann N, Pfanner N. Role of MINOS in mitochondrial

19 membrane architecture and biogenesis. Trends Cell Biol. 2012;22(4):185-92. Epub 2012/03/06. doi:

20 10.1016/j.tcb.2012.01.004. PubMed PMID: 22386790.

21 73. Aaltonen MJ, Friedman JR, Osman C, Salin B, di Rago JP, Nunnari J, et al. MICOS and

22 phospholipid transfer by Ups2-Mdm35 organize membrane lipid synthesis in mitochondria. J Cell Biol.

23 2016;213(5):525-34. Epub 2016/06/01. doi: 10.1083/jcb.201602007. PubMed PMID: 27241913; PubMed

24 Central PMCID: PMCPMC4896057.

25 74. Chojnacka M, Gornicka A, Oeljeklaus S, Warscheid B, Chacinska A. Cox17 Protein Is an 26 Auxiliary Factor Involved in the Control of the Mitochondrial Contact Site and Cristae Organizing 
1 System. J Biol Chem. 2015;290(24):15304-12. Epub 2015/04/29. doi: 10.1074/jbc.M115.645069.

2 PubMed PMID: 25918166; PubMed Central PMCID: PMCPMC4463469.

3 75. Alkhaja AK, Jans DC, Nikolov M, Vukotic M, Lytovchenko O, Ludewig F, et al. MINOS1 is a

4 conserved component of mitofilin complexes and required for mitochondrial function and cristae

5 organization. Mol Biol Cell. 2012;23(2):247-57. Epub 2011/11/25. doi: 10.1091/mbc.E11-09-0774.

6 PubMed PMID: 22114354; PubMed Central PMCID: PMCPMC3258170.

7 76. Nezich CL, Wang C, Fogel AI, Youle RJ. MiT/TFE transcription factors are activated during

8 mitophagy downstream of Parkin and Atg5. J Cell Biol. 2015;210(3):435-50. Epub 2015/08/05. doi:

9 10.1083/jcb.201501002. PubMed PMID: 26240184; PubMed Central PMCID: PMCPMC4523611.

10 77. McQuin C, Goodman A, Chernyshev V, Kamentsky L, Cimini BA, Karhohs KW, et al.

11 CellProfiler 3.0: Next-generation image processing for biology. PLoS Biol. 2018;16(7):e2005970. Epub

12 2018/07/04. doi: 10.1371/journal.pbio.2005970. PubMed PMID: 29969450; PubMed Central PMCID:

\section{PMCPMC6029841.}


A

\section{B}

PINK1(C125G)-EYFP stable

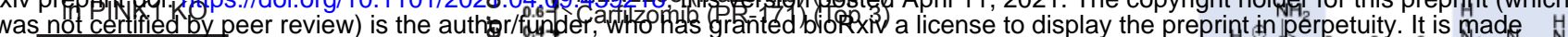
Silencer siRNA Control OMA1 (72 hour) Control OMA1

$\operatorname{CCCP}$ (4 hour) -+- -+

MG132 (4 hour) - - + - +

PINK1 (C125G) - - - Full-length

-EYFP

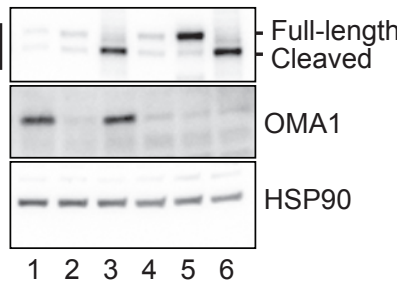

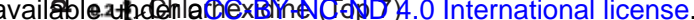

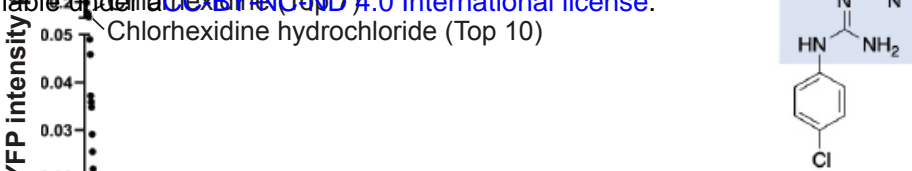

D

Compounds

$\mathbf{F}$

PINK1 (C125G)-EYFP stable in PINK1 KO

$\frac{2 \mu \mathrm{M}}{04816} \frac{5 \mu \mathrm{M}}{04816} \frac{\text { CCCP }}{\frac{20 \mu \mathrm{M}}{0816 \text { (hour) }}}$

\begin{tabular}{|l|l}
\hline \multicolumn{1}{|c|}{ Full-length | } & $\begin{array}{l}\text { PINK1 (C125G) } \\
\text {-EYFP }\end{array}$ \\
\cline { 1 - 1 } OMA1 & \\
\hline USP60 &
\end{tabular}

G

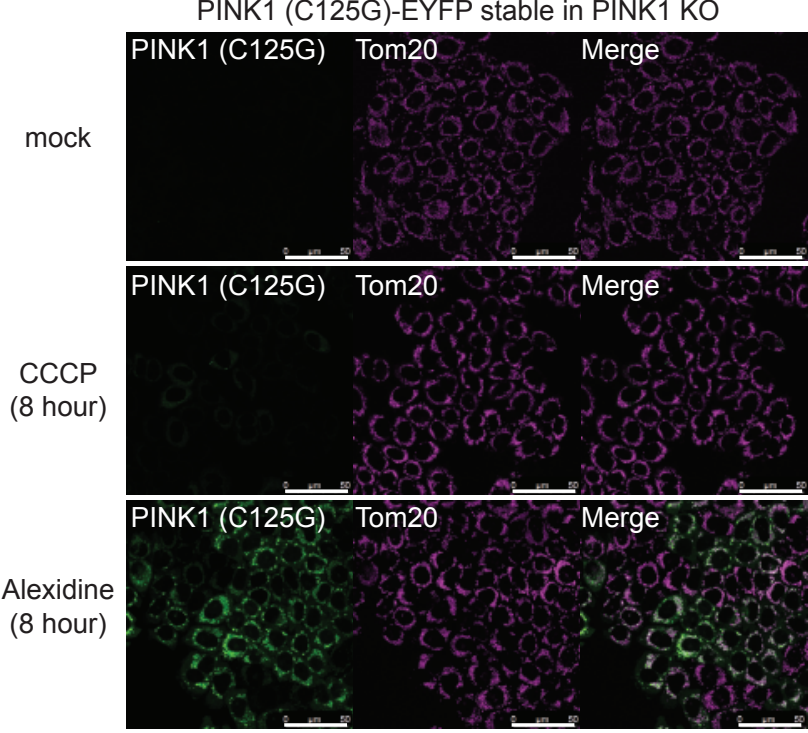

PINK1 (C125G)-EYFP stable in PINK1 KO

$\frac{\text { Alexidine }}{\frac{2 \mu \mathrm{M}}{04816} \frac{5 \mu \mathrm{M}}{04816} \frac{\mathrm{CCCP}}{20 \mu \mathrm{M}}}$

\section{Alexidine}

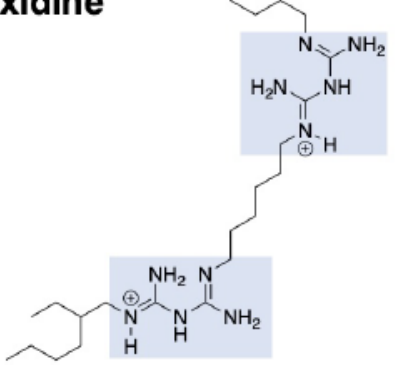

H

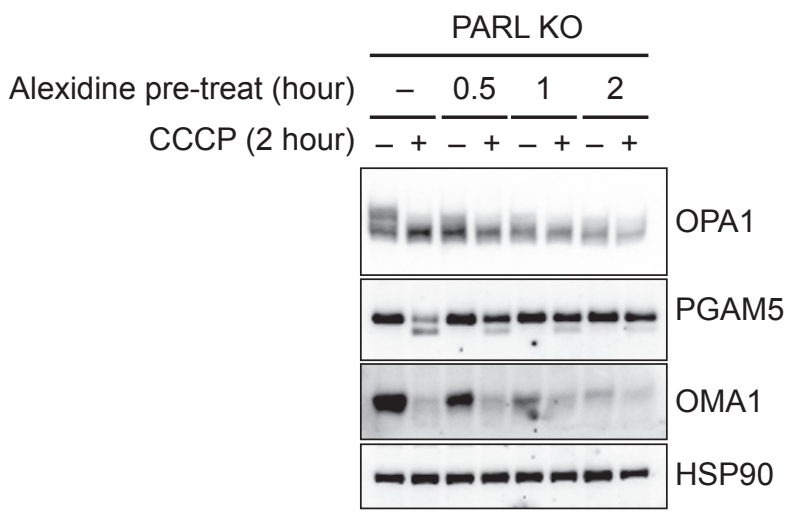

*PARL KO: OMA1-mediated PGAM5 cleavage

K

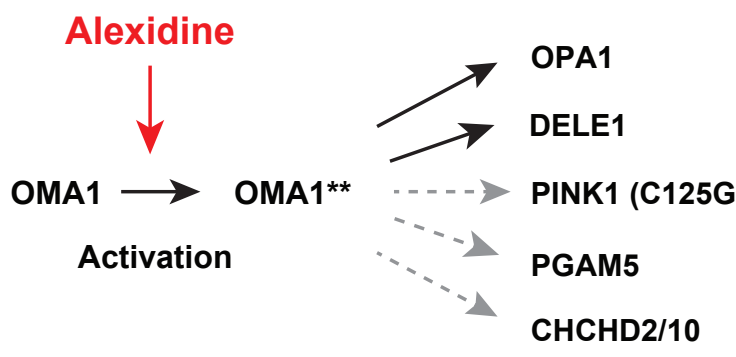

Fig. 1 Identification of bactericides as a substrate-dependent OMA1 inhibitor. 
A

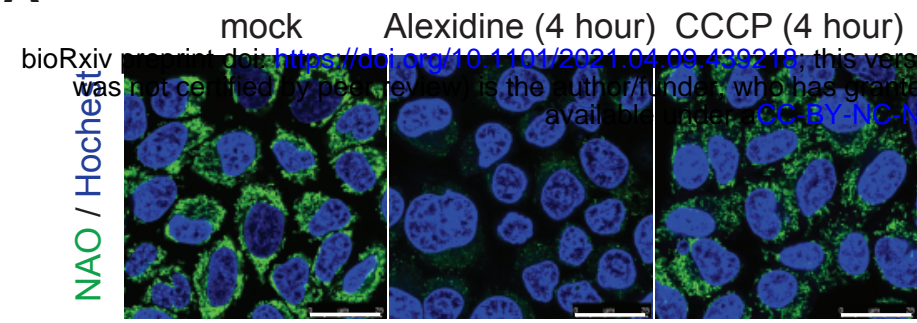

Drug treatment (4 hour) NAO staining (15 min)

Drug wash Imaging

B

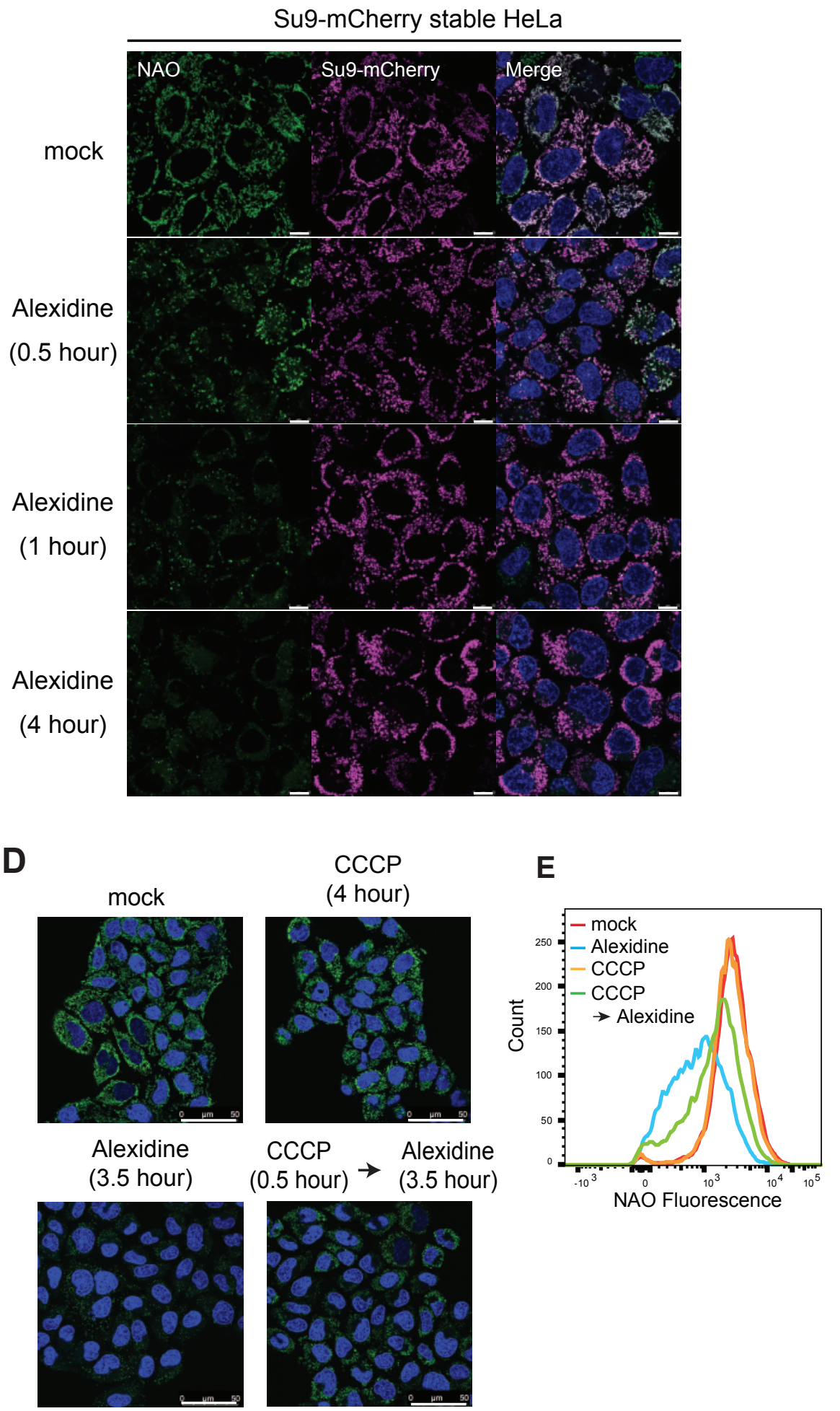

C

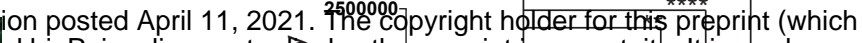
bioRxiv a license to displabotboe preprint in perpetuity. It is made D 4.0 International licerfse.
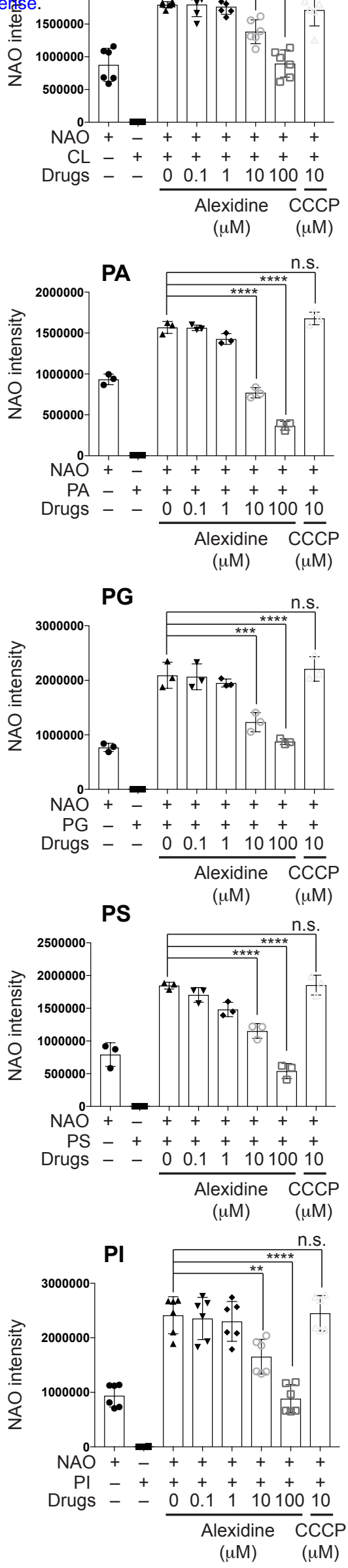

NAO / Hochest

Fig. 2 Alexidine has an affinity for the IMM. 


\section{A}
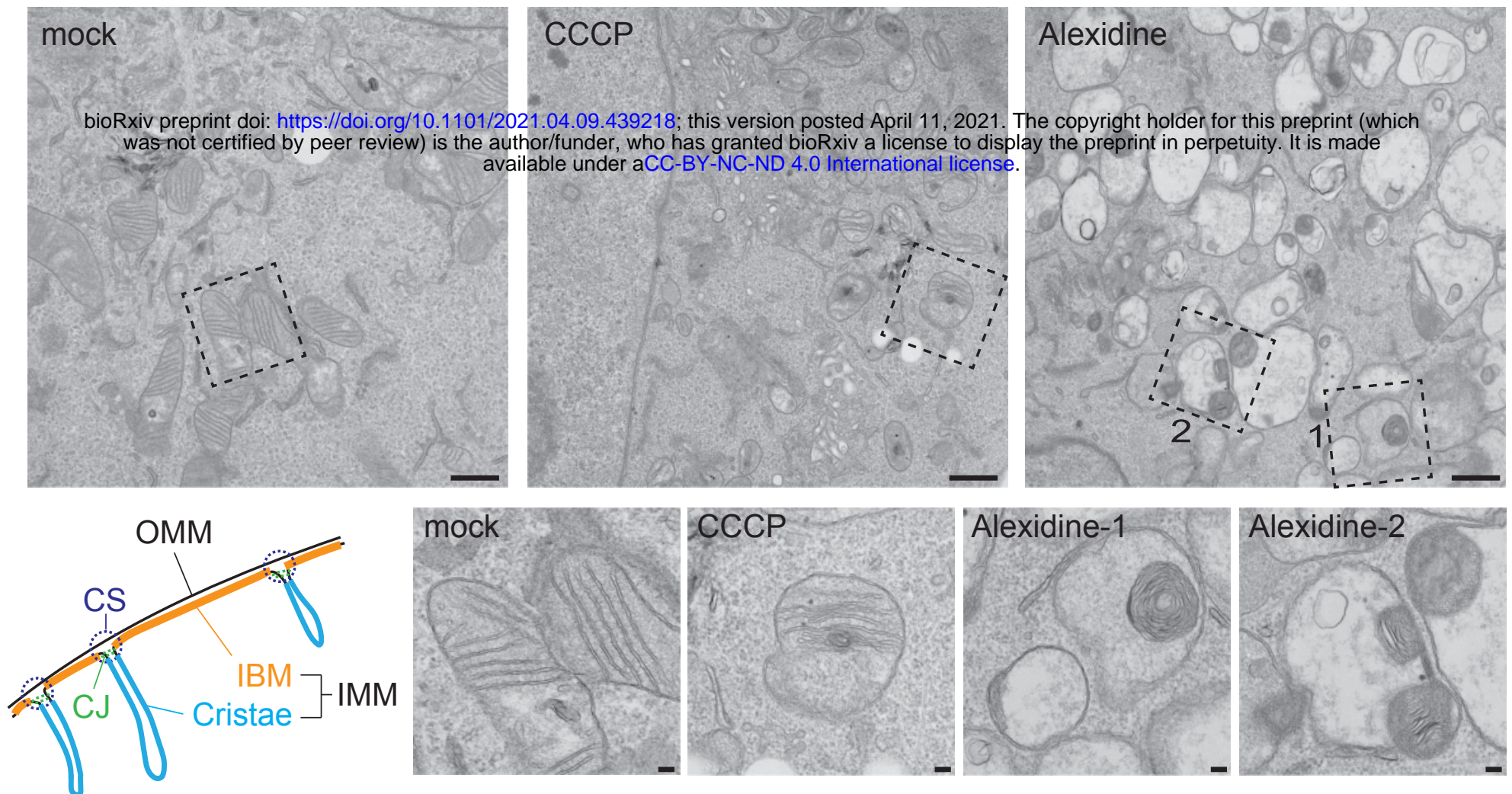

B

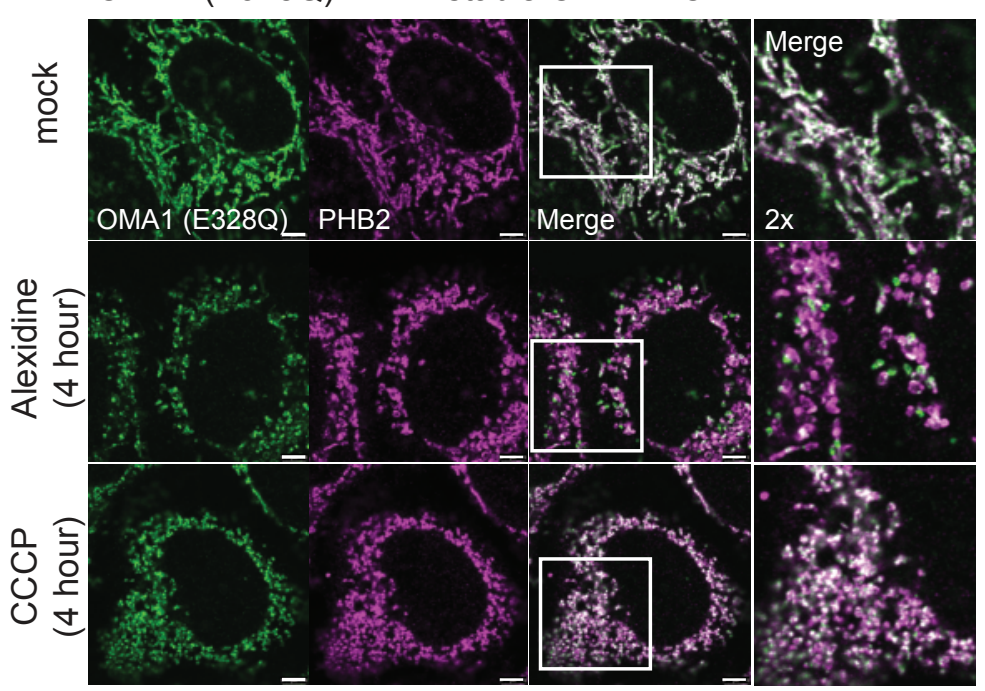

D

항

Mic60

Tim50

Merge

Oे

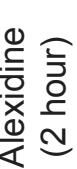

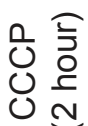

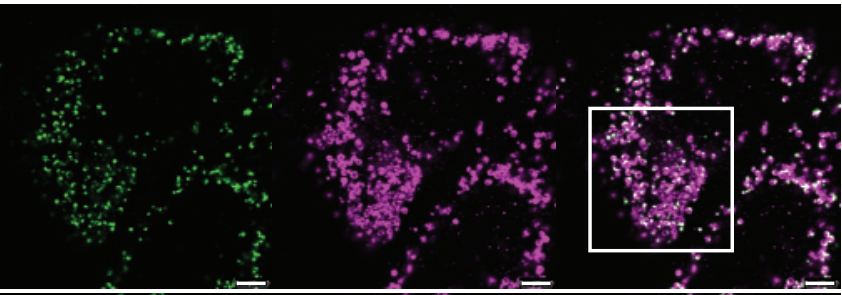

C

OMA1 (E328Q)-EYFP stable OMA1 KO
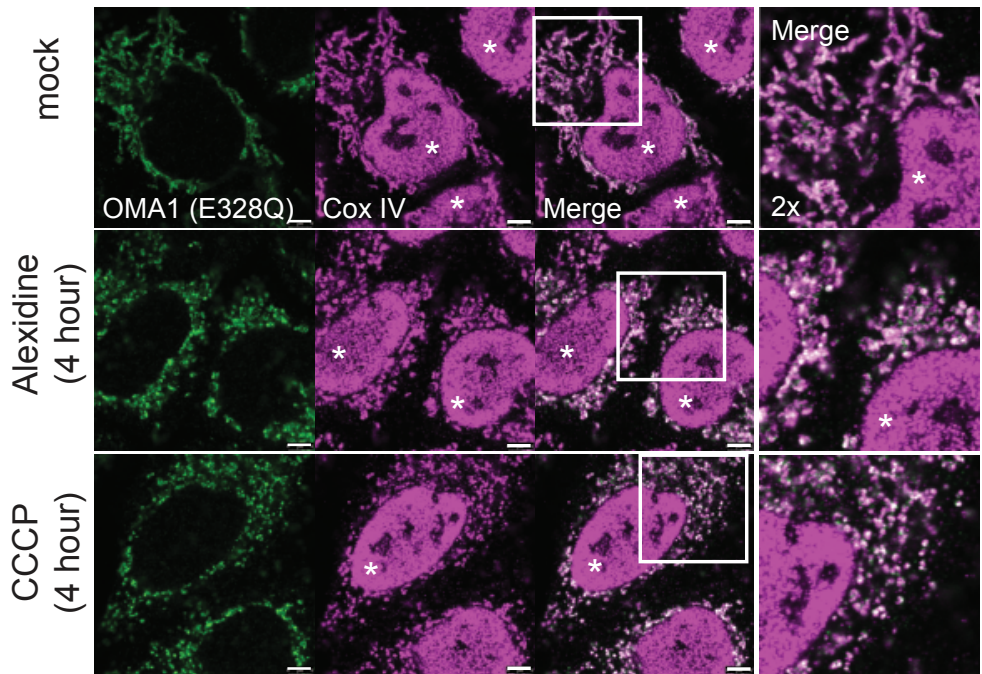

Representative images
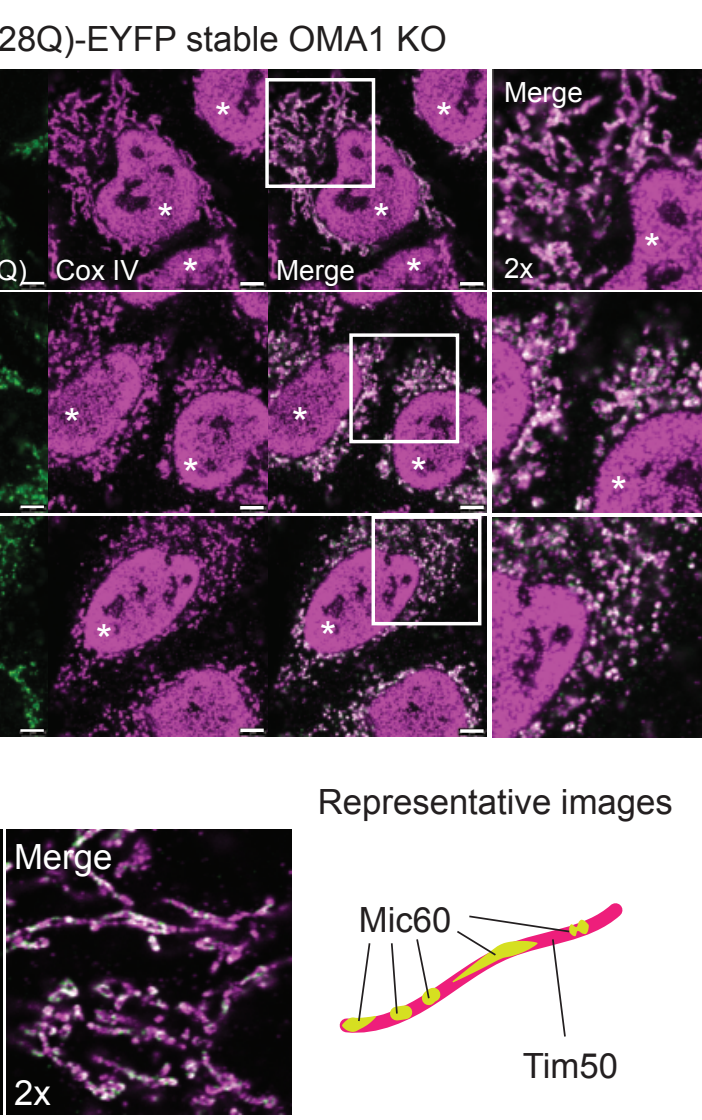

$2 x$

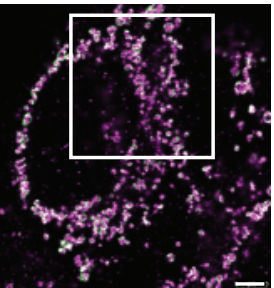

Mic60
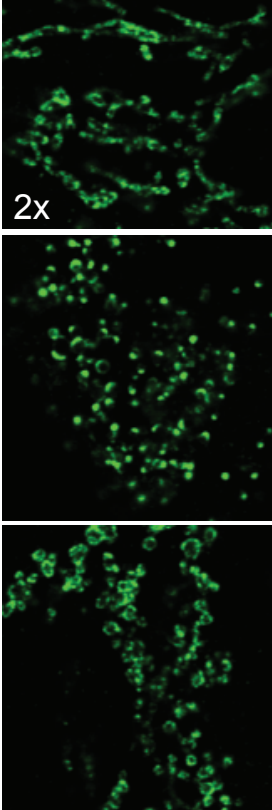

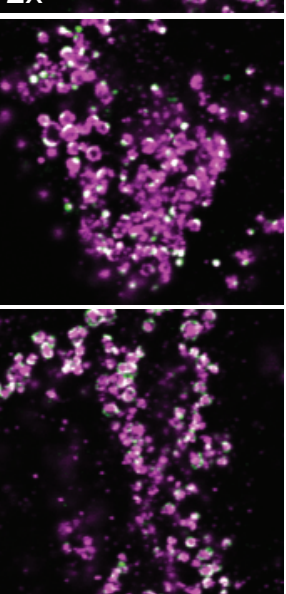

Fig. 3 Alexidine perturbates the IMM integrity. 
A

Fischer Exact test (Mock vs. Alexidine) GOCC name was not certified hry mitochondrial membrane organelle inner membrane mitochondrial inner membrane mitochondrial intermembrane space

C

Down-regulated proteins

(fold change $<0.8$, T-TEST q-value $<0.05$ )

\section{Alexidine}

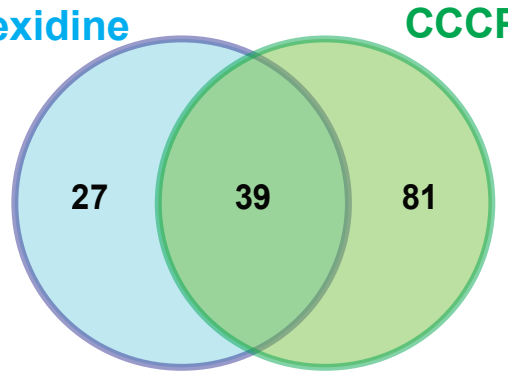

D

Alexidine-specific down-regulated mitochondrial proteins

\begin{tabular}{|c|l|l|r|l|}
\hline \multicolumn{2}{|c|}{ Biological pathways } & \multicolumn{1}{c|}{$\begin{array}{c}\text { Gene } \\
\text { names }\end{array}$} & $\begin{array}{c}\text { Fold change } \\
\text { (Mock vs. Alexidine) }\end{array}$ & Localization \\
\hline \multirow{3}{*}{ OXPHOS } & Complex I & NDUFAF4 & 0.781096009 & matrix \\
\cline { 3 - 5 } & & NDUFA8 & 0.756966902 & IMS \\
\cline { 3 - 5 } & Cytochrome C & CYCS & 0.622490602 & IMS \\
\cline { 3 - 5 } & \multirow{2}{*}{ Complex IV } & COA4 & 0.748773313 & IMS \\
\cline { 3 - 5 } & & COA6 & 0.786806125 & IMS \\
\cline { 3 - 5 } & & COA7 & 0.557477307 & IMS \\
\cline { 3 - 5 } & & COX17 & 0.710096179 & IMS \\
\hline \multirow{3}{*}{ Coenzyme Q biosynthesis } & & COQ5 & 0.789222379 & matrix \\
\hline \multirow{2}{*}{ Cardiolipin synthesis } & & COQ7 & 0.760661459 & matrix \\
\hline \multirow{2}{*}{ Others } & & PTPMT1 & 0.775402121 & matrix \\
\hline & & GLRX2 & 0.63307837 & matrix \\
\cline { 3 - 5 } & & ENDOG & 0.681358618 & IMS \\
\cline { 3 - 5 } & & PYURF & 0.755190504 & $?$ \\
\hline
\end{tabular}

TMT-proteomics (Mock vs. Alexidine vs. CCCP)

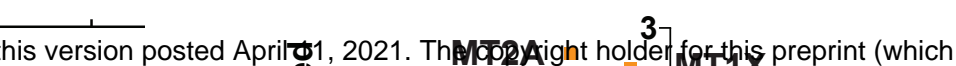
hor/funder, who has granted bioRxiv a licese to display the preprint in peffetulity. It is made

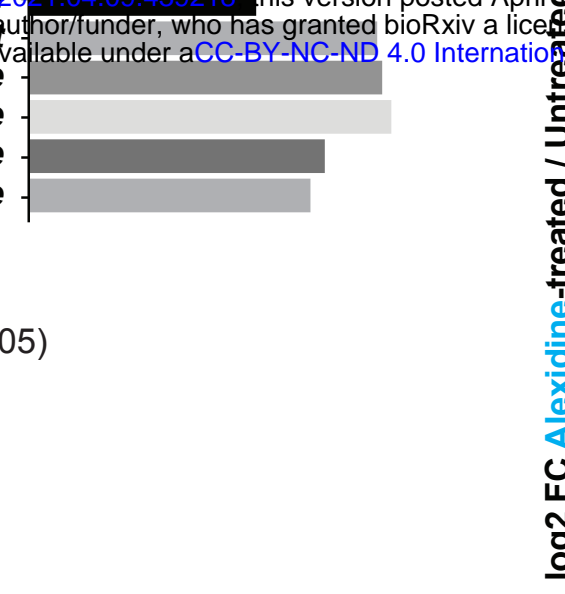

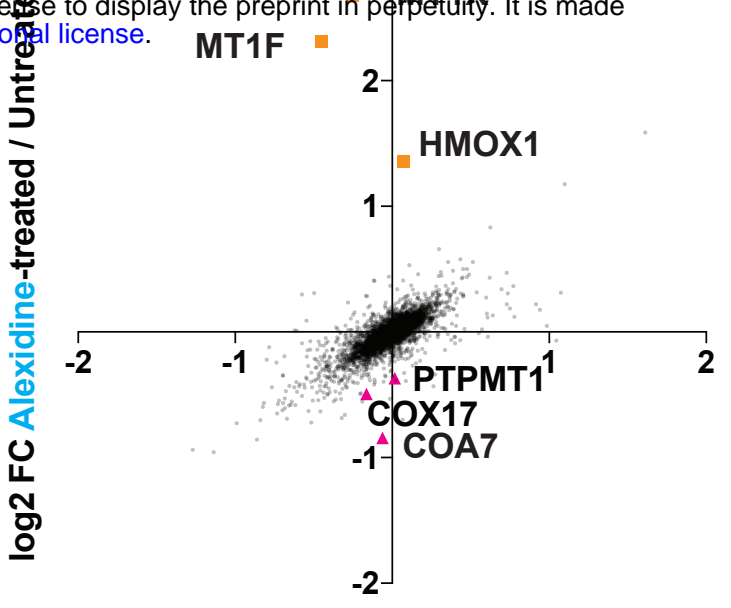

log2 FC CCCP-treated / Untreated

G

Drug treatment

(8 hour)

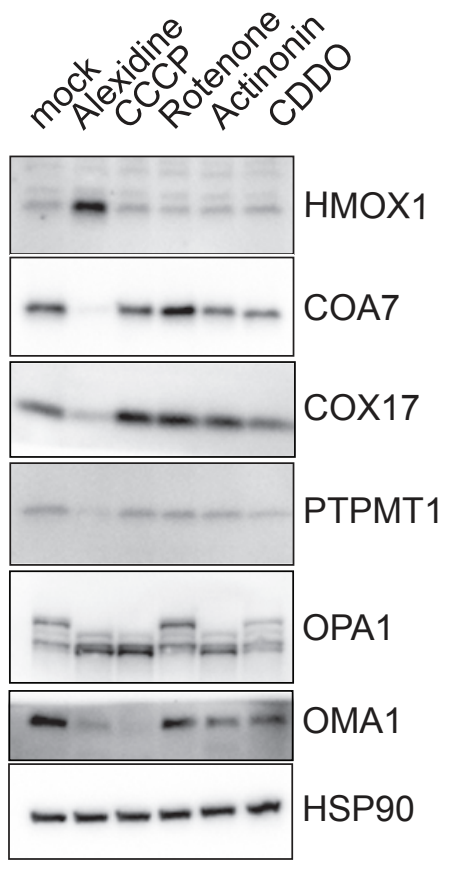

E

Up-regulated proteins

(fold change $>1.5$, T-TEST q-value $<0.05$ )

Alexidine

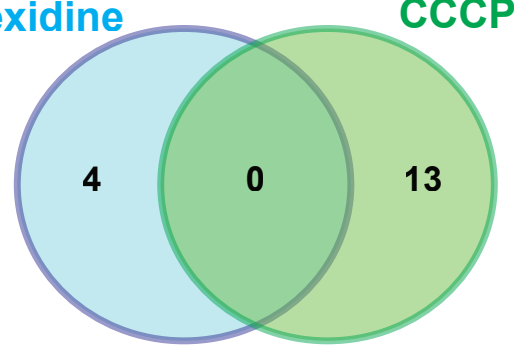

F Alexidine-specific up-regulated proteins

\begin{tabular}{|c|l|r|}
\hline Biological pathways & \multicolumn{1}{c|}{$\begin{array}{c}\text { Gene } \\
\text { names }\end{array}$} & $\begin{array}{c}\text { Fold change } \\
\text { (Mock vs. Alexidine) }\end{array}$ \\
\hline \multirow{3}{*}{ Heavy metal detoxification } & MT2A;MT1G & 6.961251437 \\
\cline { 2 - 3 } & MT1X & 6.422051265 \\
\cline { 2 - 3 } & MT1F & 4.956008461 \\
\hline Heme catabolism & HMOX1 & 2.562446302 \\
\hline
\end{tabular}

H
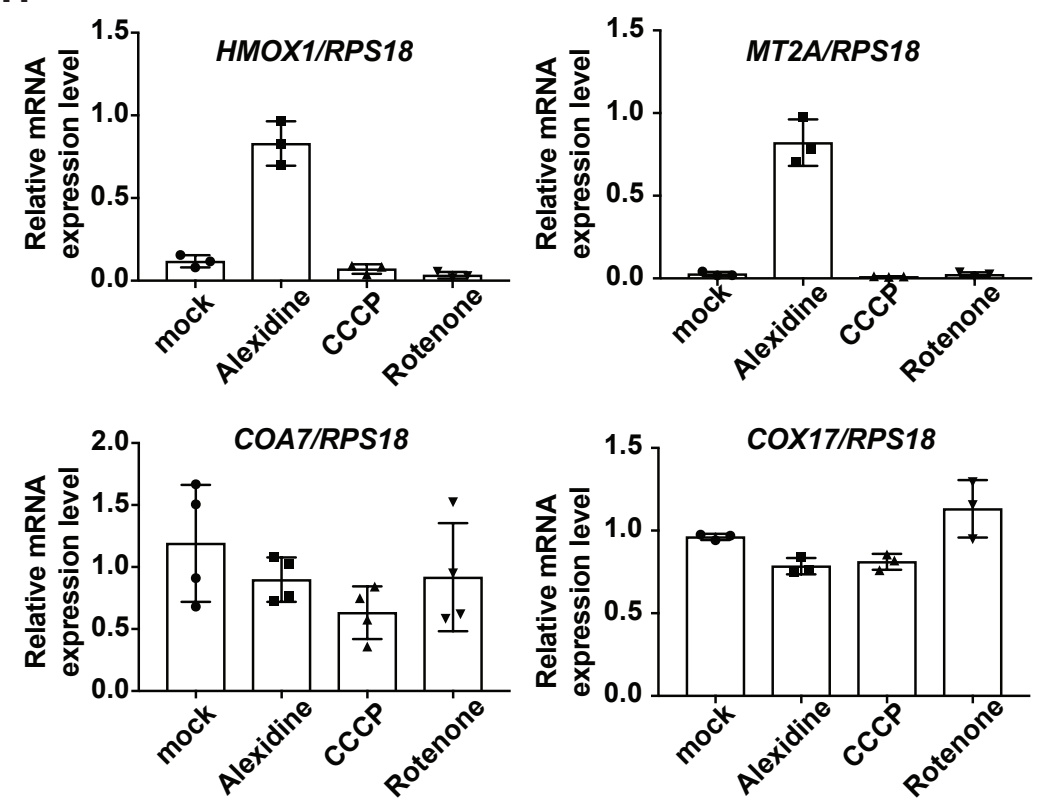

Fig. 4 Alexidine evokes a unique transcriptional/proteostatsis siganture. 
bioRxiv preprint doi: https://doi.org/10.1101/2021.04.09.439218; this version posted April 11,2021 Ghecopyright holder for this preprint (which was not certified by peer review) is the author/funder, who has granted bioRxiv a license to display the preprint in perpetuity. It is made

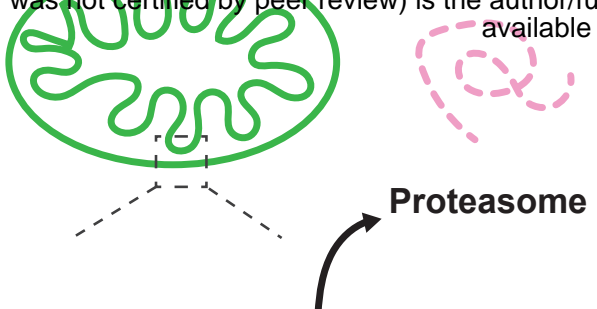

PINK1 (WT) or PINK1 (C125G)

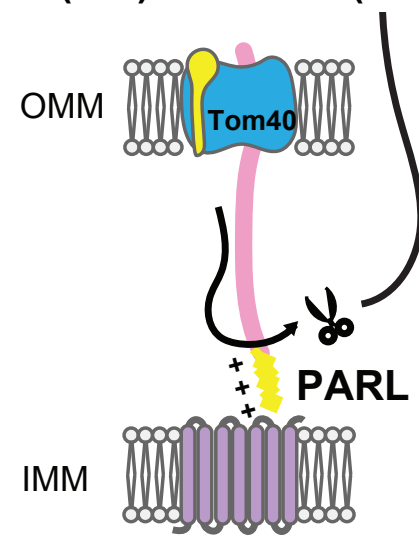

B

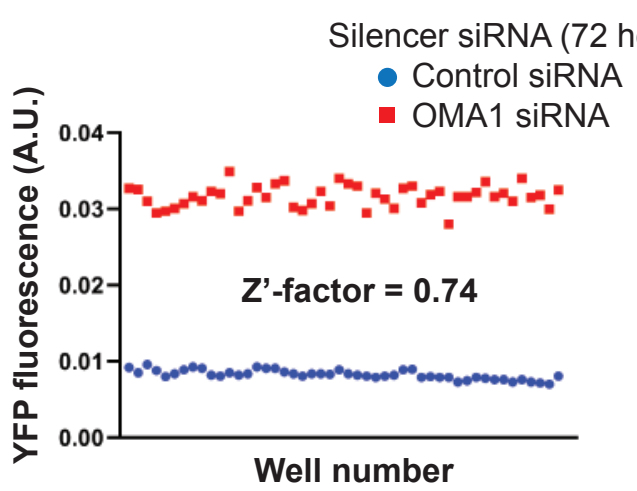

C
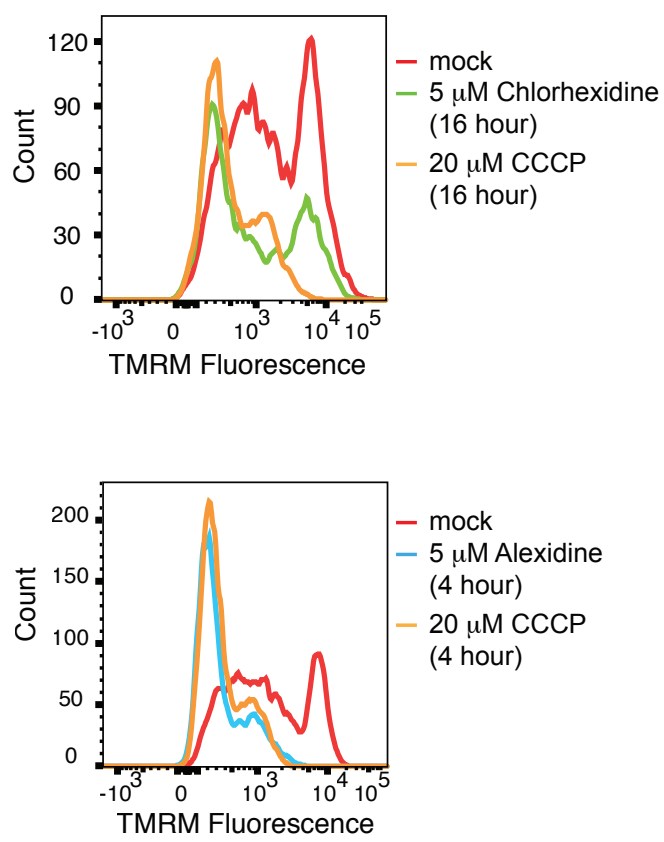

PINK1-EYFP stable in PINK1 KO

CCCP (4 hour)

PINK1 (WT)

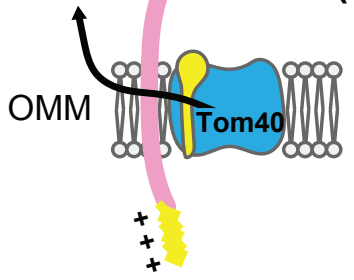

IMM

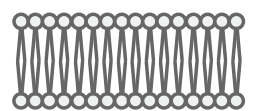

OMM

IMM

PINK1 (C125G)

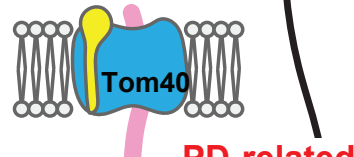

PD-related

C125G mutation

Alexidine (8 hour)

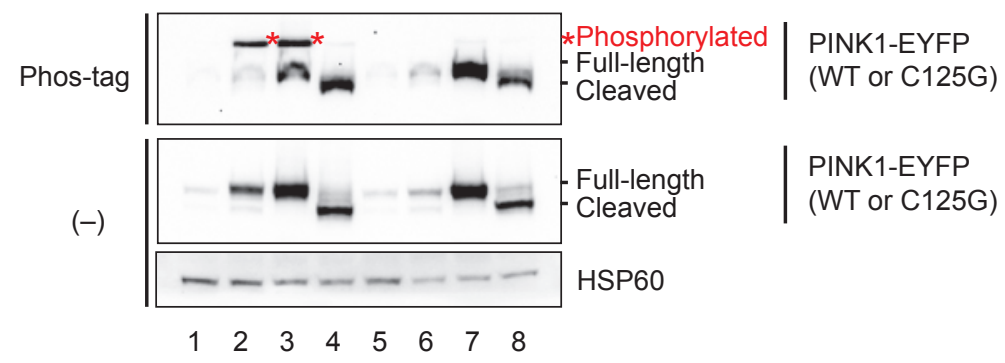

E

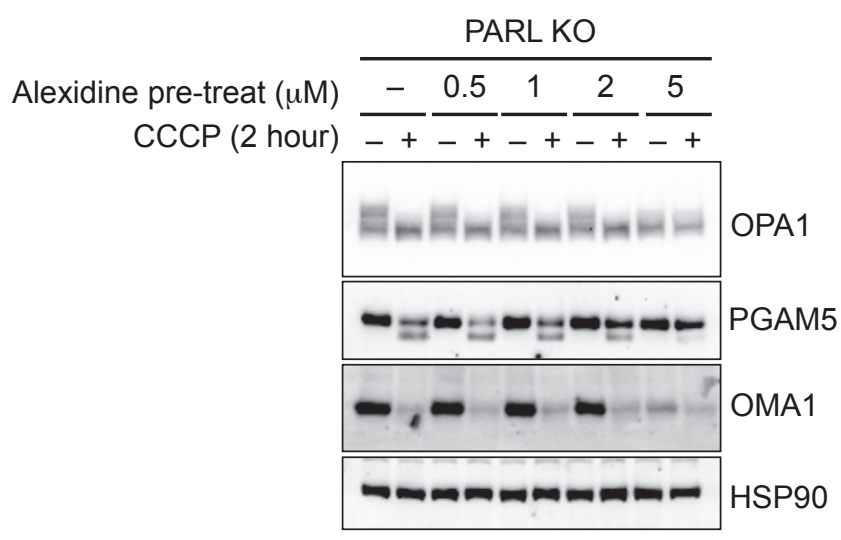

*PARL KO: OMA1-mediated PGAM5 cleavage 
bioRxiv preprint doi: https://doi.org/10.1101/2021.04.09.439218; this version posted April 11, 2021. The copyright holder for this preprint (which was not certified by peer review) is the author/funder, who has granted bioRxiv a license to display the preprint in perpetuity. It is made available under aCC-BY-NC-ND 4.0 International license.

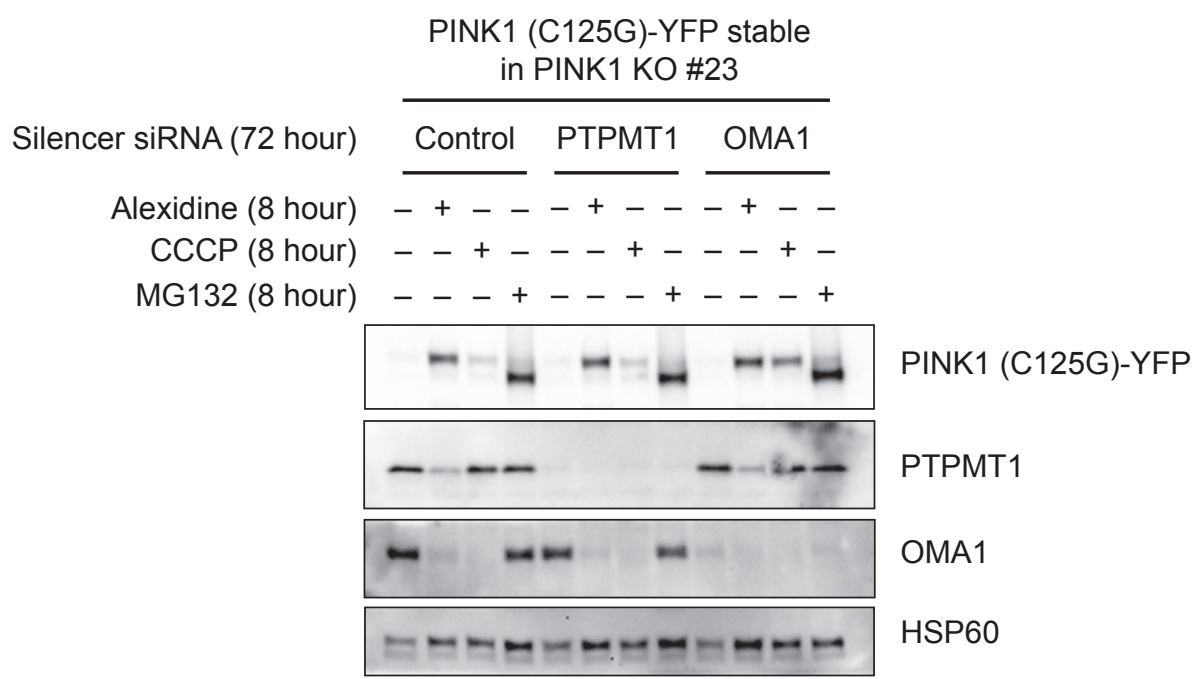

$\begin{array}{llllllllllllll}1 & 2 & 3 & 4 & 5 & 6 & 7 & 8 & 9 & 10 & 11 & 12\end{array}$

Supplementary Fig. 2

Transient deletion of PTPMT1 did not promote the accumulation of PINK1 (C125G). 
A

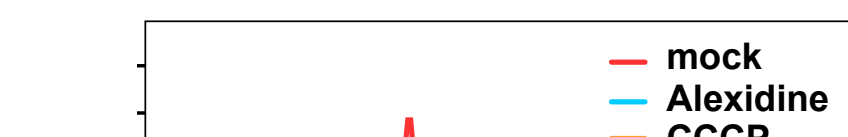

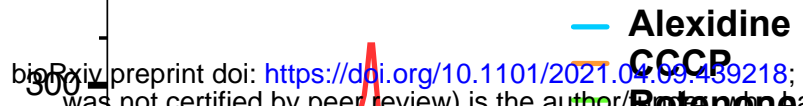

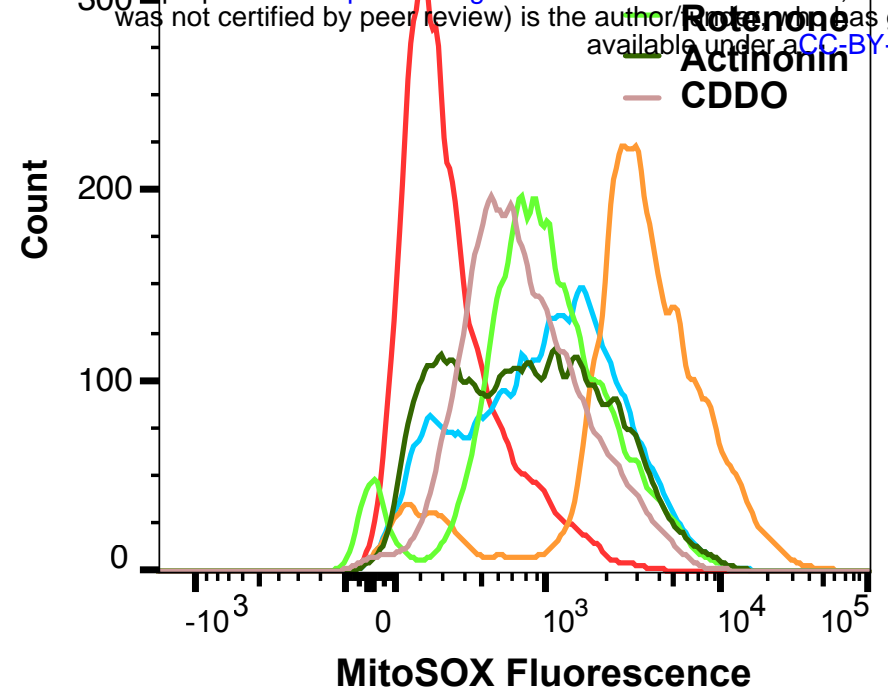

C

Silencer siRNA Stealth siRNA

(72 hour)

(72 hour)

Alexidine (8 hour)
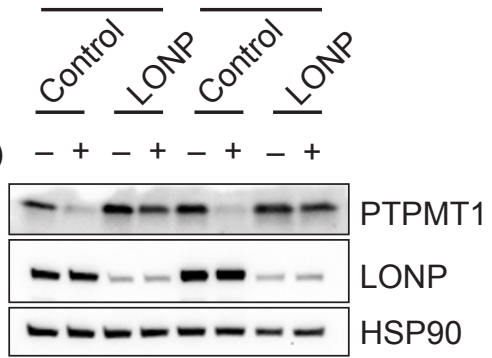

D

\section{HMOX1, MTs}
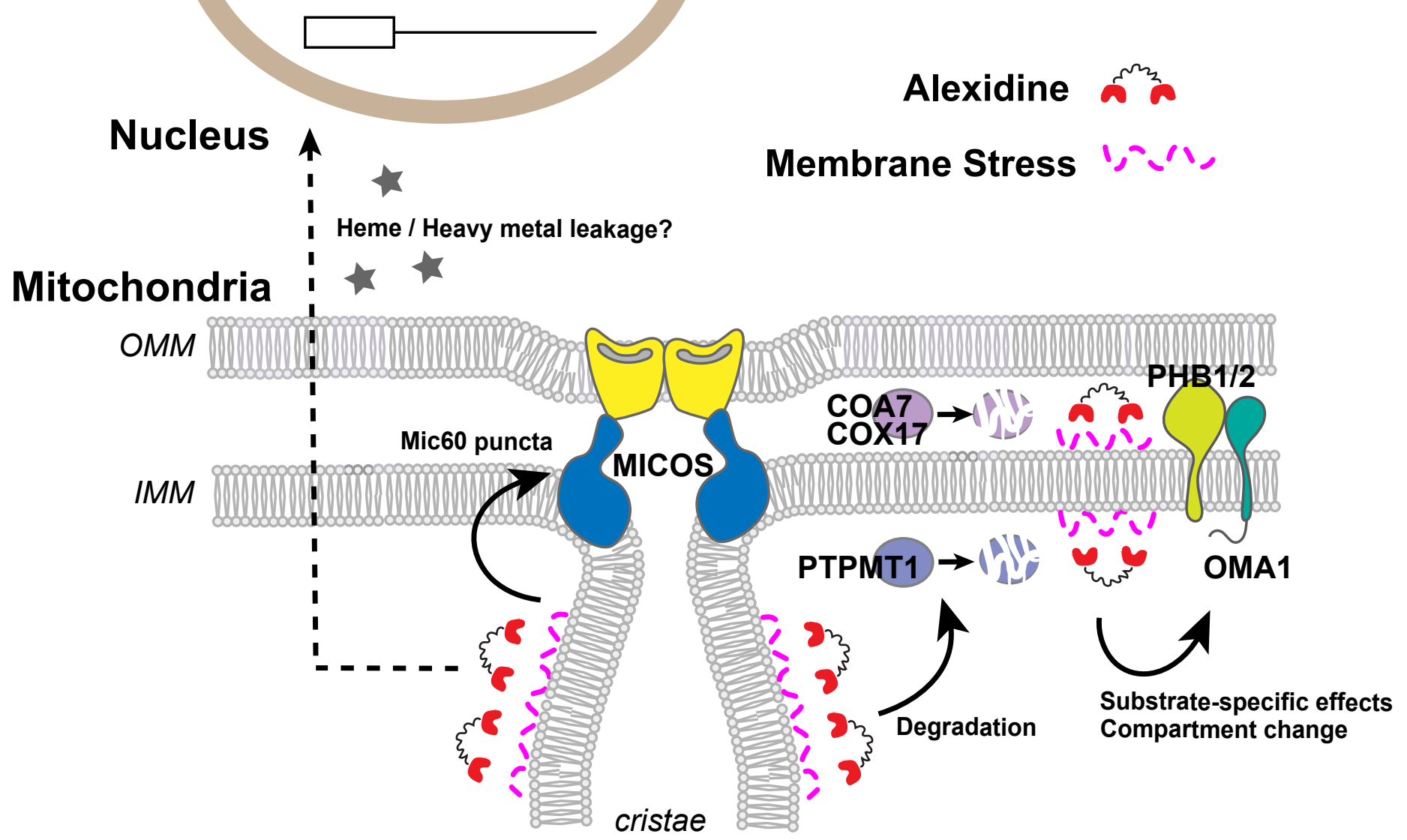

Supplementary Fig. 3

Alexidine is a novel mitochondrial stressor that can induce the acute mitochondrial membrane damage. 